This is a self-archived version of an original article. This version may differ from the original in pagination and typographic details.

Author(s): Aunola, Kaisa; Sorkkila, Matilda; Tolvanen, Asko; Tassoul, Alexandra; Mikolajczak,
Moïra; Roskam, Isabelle

Title: Development and validation of the Brief Parental Burnout Scale (BPBS)

Year: 2021

Version: Accepted version (Final draft)

Copyright: 두 2021 American Psychological Association

Rights: In Copyright

Rights url: http://rightsstatements.org/page/InC/1.0/?language=en

Please cite the original version:

Aunola, K., Sorkkila, M., Tolvanen, A., Tassoul, A., Mikolajczak, M., \& Roskam, I. (2021). Development and validation of the Brief Parental Burnout Scale (BPBS). Psychological Assessment, 33(11), 1125-1137. https://doi.org/10.1037/pas0001064 
Running head: BRIEF PARENTAL BURNOUT SCALE

\title{
Development and Validation of the Brief Parental Burnout Scale (BPBs)
}

\author{
Kaisa Aunola ${ }^{1}$, Matilda Sorkkila ${ }^{1}$, Asko Tolvanen ${ }^{2}$, Alexandra Tassoul $^{3}$, \\ Moïra Mikolajczak ${ }^{3}$, and Isabelle Roskam ${ }^{3}$ \\ ${ }^{1}$ Department of Psychology, University of Jyväskylä, Finland \\ ${ }^{2}$ Methodology Centre for Human Sciences, University of Jyväskylä, Finland \\ ${ }^{3}$ Department of Psychology, Catholic University of Louvain (UCLouvain), Belgium
}

In Press: Psychological Assessment

(c) 2021, American Psychological Association. This paper is not the copy of record and may not exactly replicate the final, authoritative version of the article. Please do not copy or cite without authors' permission. The final article will be available, upon publication, via its DOI:

$10.1037 /$ pas0001064

\section{Author's Note}

This study was funded by a grant from the Alli Paasikivi Foundation (grant number 21000041571) to Kaisa Aunola. We have no conflicts of interest to disclose.

This study was not preregistered. The data, study materials, and analysis code used in this study are available from the first author upon reasoned request.

Correspondence concerning this article should be addressed to Kaisa Aunola, Department of Psychology, University of Jyväskylä, P.O. BoX 35, 40014 University of Jyväskylä, Finland; Email: kaisa.aunola@jyu.fi 


\begin{abstract}
Since parental burnout can have detrimental consequences for parents, couples, and children, easy-to-use, reliable, and valid practical tools for the early detection of parental burnout are required. We developed and validated a brief scale to identify burned-out parents and parents at burnout risk. In Study 1, we developed the 5-item Brief Parental Burnout scale (BPBs) based on the gold standard measure of parental burnout, the Parental Burnout Assessment
\end{abstract} (PBA). We applied Item Response Theory Graded Response Model (GRM) analyses on the data of 1,725 Finnish parents. Compared against the PBA total score, the five selected items showed both high sensitivity and specificity in screening parental burnout. As expected, burned-out parents and those at burnout risk according to the BPBs displayed higher depressive symptoms, lower self-esteem, and more frequent sleep disruptions than nonburned-out parents. In Study 2, we tested the sensitivity and specificity of the BPBs by using both the BPBs and the PBA as independent assessment tools in a sample of 1,088 Finnish parents. In Study 3, the high sensitivity and specificity of the BPBs found in Study 2 were replicated in an independent sample of 104 Belgian parents. We further demonstrated the concurrent validity of the BPBs test score interpretations by showing that burned-out parents, or those at burnout risk, reported higher child neglect and parental violence than non-burnedout parents. Because of its user-friendliness and the strong psychometric properties of its test score, the BPBs is a good candidate to use as a screening tool for parental burnout.

Keywords: exhaustion, parenting, screening tool, sensitivity, specificity

Public Significance Statement: We developed an easy-to-use, practical screening tool for the early detection of parental burnout and the risk of parental burnout. Due to its strong psychometric properties and user-friendliness, we suggest the screening tool be systematically implemented in health care services. 


\section{Development and Validation of the Brief Parental Burnout Scale (BPBs)}

Although parenting can be fulfilling and joyful, it can simultaneously be characterized as stressful and demanding (Crnic \& Low, 2002). When parental demands constantly exceed the available parental resources, the parent may be at risk of burnout (Mikolajczak \& Roskam, 2018). Parental burnout is a syndrome characterized by exhaustion in one's parental role, feeling fed up with being a parent, emotional distancing from one's children, and contrast with one's previous parental self (Roskam et al., 2018). Since parental burnout can have detrimental consequences for parents (e.g., escapism and suicidal thoughts), couples (e.g., couple conflicts), and children (e.g., neglectful and violent behavior towards children) (Mikolajczak et al., 2018; Mikolajczak et al., 2019), it is essential to generate easy-to-use, reliable, and valid practical tools for the early detection of parental burnout. The purpose of this study was to develop and validate a short screening tool to identify either burned-out parents or parents at burnout risk. Based on the gold standard measure of parental burnout, the Parental Burnout Assessment (PBA, Roskam et al., 2018), we generated the Brief Parental Burnout scale (BPBs), a short 5-item screening tool. It was designed to be easy-touse by health care staff outside the research community (e.g., nurses). This type of measure is designed to enable the early detection of parental burnout through, for example, vast countrywide standardized screenings with the goal of intervention aimed at preventing it.

\section{Parental Burnout}

Although burnout has traditionally been studied in the occupational context (Maslach et al., 2001), it has been recently recognized that burnout can take place in any context that is characterized by chronic stress (Bianchi et al., 2014). One context that can be particularly stressful is parenting (Crnic \& Low, 2002; Deater-Deckard, 1998). Parenting includes managing daily tasks (e.g., cooking, cleaning), as well as dealing with acute stressors (e.g., sibling conflicts, sick children) and sometimes chronic stressors (e.g., lack of sleep, 
children's special needs) (Mikolajczak et al., 2019). Whereas work duties are usually executed 8-10 hours per day, followed by free time and recovery, parenting duties can last 24 hours per day, particularly when children are young (Deater-Deckard, 1998) or when adolescents are difficult to handle. Furthermore, whereas it is possible to quit work when facing a chronically too high workload, it is impossible for parents to resign.

Parental burnout has been shown to differ from job burnout and depressive symptoms by both factorial distinctiveness and consequence distinctiveness (Mikolajczak et al., 2020). Specifically, parental burnout, job burnout, and depression have been shown to load consistently on different latent variables (Mikolajczak et al., 2020). Also, compared to depression, parental burnout and job burnout tend to affect more specifically the context of life from which they originate (e.g., parental burnout is associated with parental satisfaction; job burnout is associated with job satisfaction), whereas depression is more all-pervasive (Mikolajczak et al., 2020). For example, parental burnout seems to have specific parentingrelated consequences (Mikolajczak et al., 2019; Mikolajczak et al., 2020): whereas job burnout explains less than $1 \%$ of the variance of child neglect and parental violence towards children, parental burnout explains up to 31\% (Mikolajczak et al., 2018).

Recent evidence suggests that the four core symptoms of parental burnout, that is, exhaustion in one's parental role, feeling fed up with being a parent, emotional distancing from one's children, and contrast with one's previous parental self, do not appear all together at once, but rather come in stages (Roskam \& Mikolajczak, under review). Similar to the progression of both job burnout and school burnout (e.g., Parker \& Salmela-Aro, 2011; Taris et al., 2005), parental burnout has to be considered as a developmental process that starts with emotional exhaustion. Emotional exhaustion then leads to emotional distancing from one's children, which, in turn, leads to the loss of parental fulfillment (Roskam \& Mikolajczak, under review). In line with this, other recent findings have revealed that emotional exhaustion 
alone is not sufficient to turn an initially well-caring parent into a more neglectful or violent one. Rather, it is the emotional distancing that appears at a later stage that leads to child neglect and parental violence (Blanchard et al., 2020; Hansotte et al., 2021). Taken together, these results suggest that parental burnout can be detected at its early stages (i.e., when parents are at burnout risk) and that prevention-focused interventions should be implemented before the burnout has deleterious consequences.

\section{Measuring Parental Burnout}

Parental burnout measures (e.g., Parental Burnout Inventory, PBI; Roskam et al., 2017) were initially derived from the Maslach Burnout Inventory (MBI; Maslach et al., 1997), which assesses burnout in an occupational context. In the MBI, burnout is defined as a threedimensional syndrome encompassing work-related emotional exhaustion, cynicism, and decreased professional accomplishment (Maslach et al., 1997). On this basis, parental burnout was defined in the PBI as parenting-related emotional exhaustion, emotional distancing from one's children, and loss of parental accomplishment (Roskam et al., 2017). More recently, however, researchers have questioned whether the items that were derived from the work context actually best represent burnout in the parenting context (see Roskam et al., 2018). To address this problem, Roskam et al. (2018) developed the Parental Burnout Assessment (PBA). Instead of using a deductive method (i.e., items are deduced from another context), the PBA was developed inductively from interviews of burned-out parents (i.e., items are extracted from the parents' testimonies).

In the PBA, parental burnout is defined as a four-dimensional syndrome consisting of 1) exhaustion related to one's parental role (i.e., emotional depletion and chronic fatigue in one's role as a parent), 2) feelings of being fed up with one's parental role (i.e., loss of enjoyment in the time spent with one's children), 3) emotional distancing from one's children (i.e., limiting interactions to the instrumental aspects of parenting at the cost of the emotional 
aspects), and finally, 4) contrast with previous parental self (i.e., feelings of not being as good a parent as one used to be). The symptoms of parental burnout are assessed in the PBA by 23 items rated on a 7-point scale ranging from never (value 0) to daily (value 6), with the scale referring to the incidence of the assessed symptom. Thus, the maximum score on the PBA is 138, indicating that all the symptoms of burnout are evident daily. The PBA score has been shown to be a reliable and valid assessment tool in various cultural contexts (e.g., Arikan et al., 2020; Aunola et al., 2020; Chen et al., 2020; Furutani et al., 2020; Matias et al., 2020; Mousavi et al., 2020; Roskam et al., 2018; Stănculescu et al., 2020; Szczygieł et al., 2020). In addition, measurement invariance of PBA was reported across sex and 21 languages in a cross-cultural study of 42 countries (Roskam et al., 2021).

Different approaches and cut-off scores for the PBA have been suggested to identify parents with clinically significant levels of burnout, but they all lack either theoretical or empirical grounds (for a review, see Brianda et al., 2020). To remedy this problem, Brianda et al. (2020) applied a multi-informant and multi-method assessment to provide clinicians with validated cut-off scores for the PBA. By using a bundle of indicators of parental burnout, such as self-reports of parents, views of external clinical judges, and biological measures of chronic stress (e.g., the hair cortisol concentration), the researchers determined the following cut-off criteria values in the PBA: 86.26 (95\% CI: 79.49-93.03) for the identification of parents suffering from parental burnout and 52.67 (95\% CI: 40.91-64.43) for the identification of parents at risk of developing clinically significant levels of parental burnout.

Based on both its background and excellent psychometric properties of its test score, the PBA can be considered as the gold standard measure for parental burnout, i.e., the best single test that is the preferred method of diagnosing parental burnout and against which other methods of diagnosing parental burnout should be compared (for the definition of gold 
standard, see Versi, 1992). However, the 23-item measure may not be suitable for preventionfocused interventions aimed at a systematic screening of exhausted parents in health care settings. For this reason, we generated a short 5-item screening version of the PBA, called the Brief Parental Burnout scale (BPBs), to enable regular screenings of susceptive parental burnout and early preventive actions.

\section{The Current Research}

The purpose of the present study was to develop the BPBs and to validate it in two separate, independent samples. In Study 1, we first developed the BPBs, selecting the items and adapting the response scale. Second, we tested the sensitivity (i.e., the ability of the scale to correctly classify a parent as "burned-out" and needing intervention or as "at-risk" and needing prevention) and the specificity (i.e., the ability of the scale to correctly classify a parent as "non-burned-out," needing neither prevention nor intervention) of the BPBs score (BPBs referring in Study 1 into the selected items that were re-coded from original response scale of PBA into the adapted new response scale) compared against the total score of the PBA. Third, we tested the convergent validity of the test score interpretations of BPBs through its associations with relevant correlates, in particular with high levels of depressive symptoms (Mikolajczak et al.,2019; Van Bakel et al., 2018), sleep disruptions (Aunola et al., 2020; Lindström et al., 2011; Mikolajczak et al., 2018), and low levels of self-esteem or selfefficacy beliefs (Aunola et al., 2020; Lindström et al., 2011; Mikolajczak \& Roskam, 2018). In Study 2, we went a step further by using the BPBs and the PBA as two independent measures in an independent sample. Again, we tested the sensitivity and the specificity of the BPBs. In Study 3, we replicated the sensitivity and the specificity of the BPBs found in Study 2 in another independent sample and moved another step forward by testing the concurrent validity of the BPBs test score interpretations in relation to child neglect and parental violence (Mikolajczak et al., 2018, 2019). In all three studies, we complied with the APA 
ethical standards in the treatment of the samples. The study was not preregistered. The data, study materials, and analysis code used in the three studies are available from the first author upon reasoned request.

\section{Study 1}

The aim of Study 1 was to create a short, valid, and easy-to-use screening tool for parental burnout on the basis of the PBA (Roskam et al., 2018). The scale development was carried out using a sample of Finnish parents. The resulting BPBs is presented in Appendix A (translated version in English). The original version of BPBs in Finnish is presented as Supplemental material S1 and the translated version in French as Supplemental material S2.

\section{Method}

\section{Participants}

A total of 1,725 Finnish parents (91\% mothers) participated in the study. A large portion of them $(98.3 \%)$ had children living with them permanently, while the remainder had children living part-time in the household. Most of the participants were native Finnish $(99.4 \%)$ and lived in Finland (98.4\%). The age of the participants ranged between 18 and 61 $(M=36.47, S D=6.51)$. The number of children ranged from 1 to $17(M=2.11, S D=1.19)$. A total of $79.1 \%$ of the participants had at least one child under school age ( $<7$ year); $0.5 \%$ $(n=9)$ had only adult children (children ranged in age from 19 to 21, with the exception of one participant whose child was 31 years old). Seventy-nine percent of the parents lived in a nuclear family (of which $0.5 \%$ were same-sex families), $10 \%$ lived in a single-parent household, and 9\% lived in a blended family. Seventy-four percent had a university or college degree, $8 \%$ had a technical college degree, $15 \%$ had a vocational school degree, and $3 \%$ had no vocational degree. The highly educated parents (i.e., university or college degree) were over-represented in the sample (in the Official Statistics of Finland, 2017, the percentage of parents in Finland with a university or college degree was 44\%). 


\section{Procedure}

The study was conducted as part of the International Investigation of Parental Burnout (IIPB), a consortium involving 42 countries worldwide (Roskam et al., 2021). The data was collected from the beginning of March to the end of April in 2018. Parents were eligible to participate in the study only if they had at least one child still living at home. The informed consent they signed allowed the participants to withdraw at any stage without having to justify their withdrawal. The participants were assured that the data would remain anonymous.

Before collecting the data, ethical permission for the study was obtained from the ethical committee of the relevant university. All the participants provided informed consent to confirm their voluntary participation in the study. The parents completed either a pen-andpaper questionnaire at child health centers located in three Finnish cities (13\%) and at congregational family playgroups located in one Finnish city (1\%) or an online questionnaire advertised through different social media channels $(86 \%)$. The child health centers were selected based on geographical representativeness (the cities represent southern, middle, and northern Finland) and were considered appropriate locations for reaching a heterogeneous sample of parents (e.g., including different family types from different socioeconomic classes), as all Finnish parents are required to take their children aged 0-6 for annual checkups at these centers. Due to the small number of participating fathers, two family playgroups were also included that had specific playgroups for fathers and children. The online questionnaires were used because they enabled us to reach a large number of parents from different areas of Finland.

Whatever the questionnaire format, the parents received the same information and consent form before starting the survey. In the child health centers, the nurses were instructed to give the questionnaires to the parents at the end of their children's annual check-up. The 
nurses asked them to read the instructions and to complete the questionnaire in the waiting room and then drop it anonymously into a post box marked with the project's name.

Alternatively, the parents were given the option to take the questionnaire home and send it back to the researchers anonymously in a pre-paid envelope. In the family playgroups, the playgroup leaders gave the questionnaires to the parents when the playgroups ended and asked them to read the instructions and to complete the questionnaire and return it to the researchers anonymously in a pre-paid envelope.

\section{Measures}

Parental burnout. Parental burnout was measured using the Finnish version (Aunola et al., 2020) of the PBA (Roskam et al., 2018). The scale consists of 23 items; nine measure exhaustion in one's parental role (e.g., I feel completely run down by my role as a parent), six measure the contrast with the previous parental self (e.g., I don't think I'm the good father/mother that I used to be to my children), five measure feelings of being fed up as a parent (e.g., I can't stand my role as father/mother anymore), and three measure emotional distancing from one's children (e.g., I do what I'm supposed to do for my children but nothing more). All items were rated on a 7-point Likert scale indicating how often the parent feels a certain way $(0=$ never; $6=$ every day $)$. The Finnish version of the PBA has been shown to demonstrate good internal consistency, with Cronbach's alpha reliabilities of .97 for the total score, and $.93, .93, .90$, and .81 for the scores of four subscales, respectively, and the correlations between the four factors varying from .66 (exhaustion and emotional distancing) to .78 (contrast with previous parental self and feelings of being fed up as a parent) (Aunola et al., 2020).

Depressive symptoms. Depressive symptoms were measured using six items (e.g., I often feel sad; I am less interested in other people than before) from the revised version of Beck's Depression Inventory (BDI; Beck et al., 1961). Parents rated the items on a 5-point 
Likert scale $(1=$ not at all true of me $5=$ completely true of $m e)$. The Cronbach's alpha reliability for the total score in the present study was .86 .

Self-esteem. Self-esteem was assessed using four items (e.g., I take a positive attitude toward myself; I am able to do things as well as most other people) drawn from the Rosenberg Self-Esteem Scale (Rosenberg, 1979). The items were rated on a 5-point Likert scale $(1=$ not at all true of me; $5=$ completely true of $m e)$. The Cronbach's alpha reliability for the total score of four-items was .80 .

Sleep disruptions. Parents' sleep disruptions were measured using three dichotomous questions: "Do you feel that you get enough sleep?", "Can you easily fall asleep in the evenings?", and "Do you often wake up at night and cannot get back to sleep again?" (Reversed item). Parents answered either "yes" (value 1) or "no" (value 2).

\section{Analysis Strategy}

First, in order to identify the most optimal items to include in the short screening tool, a Item Response Theory (IRT) Graded Response model (GRM) analyses for all 23 items of the PBA was conducted using the Mplus statistical program (Muthén \& Muthén, 1998-2019), with the maximum likelihood robust (MLR) estimation method. In the present study, the item information functions from the GRM were used to find out which PBA items function best for screening purposes. The results of the GRM analyses were processed keeping in mind the following statistical criteria (see also, Olino et al., 2012). The items selected for the brief scale should demonstrate high discrimination between parents particularly in higher half of the latent parental burnout continuum (i.e., discriminate burned-out parents and those at burnout risk from those who are non-burned- out). Besides, to maximize information the selected items should represent different endpoints of the item distribution on the region under interest by demonstrating different levels of item difficulty (or, as in the case of the present study, item severity). In the current study, item difficulty describes where the item 
functions along the parental burnout continuum (e.g., an "easy" item works well among the parents showing less severe burnout symptoms, whereas a "hard" item works well among parents with severe burnout symptoms). Overall, by using both item discrimination and difficulty parameters in the item selection, it was possible to specify how accurately a particular item estimated parental burnout for different burnout levels. Because in the present study we were developing a screening tool that discriminates burned-out parents and parents at burnout risk from those who are non-burned-out, the measurement precision in the higher half of the latent trait continuum (about $1 S D$ or higher above the mean) was under focus in particular.

Beside these statistical criteria, the results of the GRM analyses were processed keeping in mind the following preconditions and content criteria. To be useful in practice, the brief scale should not include more than five items. Also, the selected items should not be threatening or too personal, so that the parents would feel free to answer honestly, even in non-anonymous situations (i.e., health care settings). Also, the content of the selected items should be distinct enough from each other to avoid redundancy. Finally, although the theoretical four-factor model of the PBA has been shown to fit empirical data, intercorrelations between the four factors were high (e.g., Aunola et al., 2020; Roskam et al., 2018). This suggests that the primary focus of the brief scale should be to find the optimal items among the 23 original ones and, only secondarily, to ensure the representativeness of the four factors.

Second, the response scale for the brief scale was created using the content criteria. For the screening purpose, the response scale should be easy to answer by including a limited number of response possibilities. We opted for a 3-point frequency scale, that is, daily (the response option 6 in the original PBA), once or twice a week (response options 5 and 4 in the original PBA), and more seldom/never (response options 3, 2, 1 and 0 in the original PBA). 
Indeed, a dichotomized response scale (i.e., symptom evident at least once a week vs. symptom evident more seldom or never) makes it possible to distinguish at-risk and burnedout parents from non-burned-out parents, but not to distinguish burned-out parents from those who are at burnout risk. By using the content criterion instead of the statistical criterion, we avoided the possibility that the criterion would be sample-specific and the response scale sensitive to differences between populations. Finally, in order to prevent both the respondents and the test-givers from associating values to answers, we decided to use letters instead of numbers as the response possibilities in the brief scale, that is, A (daily), B (once or twice a week), and C (more seldom/never). In statistical analyses, $\mathrm{A}$ is given value 2, $\mathrm{B}$ is given value 1 , and $\mathrm{C}$ is given value 0 . In Study 1 , BPBs with this new created response scale was not yet included into data collection. Therefore, in Study 1, BPBs refers to the combination of the selected PBA items that were recoded into this new 3-point scale from 7-point response scale.

Third, we tested the sensitivity (i.e., the ability of the scale to correctly classify a parent as "burned-out" or as "at burnout risk") and the specificity (i.e., the ability of the scale to correctly classify a parent as "non-burned out") of the selected five-item combination and the re-coded response scale compared against the total score of the PBA. For these analyses, we used previously validated clinical cut-off scores of the PBA (Brianda et al., 2020), i.e., 86.26 as an indicator of a clinically significant level of parental burnout and 52.67 as an indicator of a risk to burn out.

Fourth, the three groups of burned-out, at burnout risk, and non-burned-out parents identified with the five selected items and the re-coded response scale were compared to each other according to three correlates of parental burnout, i.e., depressive symptoms, sleep disruptions, and self-esteem. The group differences in depressive symptoms and self-esteem were tested using a nonparametric bootstrap t-test, whereas those in sleep disruptions were 
tested with the $\chi^{2}$-test (cross-tabulation). The correlations between the level of parental burnout estimated with the brief scale and the three criteria variables (i.e., depressive symptoms, self-esteem, and sum score of sleep disruptions) were also calculated.

\section{Results}

\section{Choosing the Best Items for the Brief Scale}

The results of the GRM for the 23 items of the PBA are shown in Table 1 . The item characteristics and information curves of all PBA items are available as Supplemental material S3. For the PBA items (see Table 1), discrimination parameter ranged from 1.43 (item ED1) to 3.69 (item EX2) and, thus, discrimination was high (1.35 - 1.69) or very high (> 1.70)(Baker, 2001) in the case of all 23 items. The six difficulty parameters from the first to the sixth threshold ranged from -1.13 to $0.71,-0.20$ to $1.44,0.43$ to $1.97,0.96$ to 2.47 , 1.30 to 2.86 , and 2.04 to 3.75 , respectively.

Eight of the items (see Table 1) were not considered suitable from content point of view and, consequently, the discrimination and difficulty parameters of them were not examined further when selecting suitable items for the brief scale. From the remaining 15 items, 7 represented exhaustion (EX) subscale of PBA, 4 contrast for previous parental self (CO), 3 emotional distancing (ED), and 1 feelings of being fed up as a parent (FU). Based on item difficulty (i.e., severity) parameters, items ED1 and ED3 were associated with a high severity level of parental burnout, whereas items ED2, CO1, CO6, CO5, FU3, EX2 and EX5 were related with moderate level of severity, and EX1, EX3, EX4, EX7, EX8, and CO4 with the lowest levels of severity. ${ }^{1}$ From these 15 items, we first identified three to be considered for

\footnotetext{
${ }^{1}$ The identified severity levels of the items representing different subscales were in line with the recent findings concerning the developmental order of different symptoms of parental burnout (Roskam \& Mikolajczak, under review), which results suggest that parental burnout is a developmental process that starts with emotional exhaustion (emotional exhaustion being thus less severe symptom) and leads gradually to emotional distancing from one's children and the loss of parental fulfillment (emotional distancing and the loss of parental fulfillment being more severe symptoms).
} 
the brief scale. They represented different endpoints of the item distribution and were suitable from the content point of view. These items were EX8 ["I sometimes have the impression that I'm looking after my child(ren) on autopilot"] (the lowest item difficulty), ED3 ["I'm no longer able to show my child(ren) how much I love them"] (the highest item difficulty and the highest item discrimination among high difficulty items); and EX2 ["I have the sense that I'm really worn out as a parent"] (medium item difficulty and the best item discrimination).

Then, from the 12 remaining items two were selected, EX3 ["I'm so tired out by my role as a parent that sleeping doesn't seem like enough"] and FU3 [“I feel like I can't take any more as a parent"], because these items (a) showed very high discrimination ability, (b) represented different levels of item difficulty (low and medium, respectively), and (c) were easy to answer and not redundant with the other three selected items (i.e., demonstrated minimal content overlap). The final item selection for the BPBs represented three out of the initial four factors of the PBA. When using the original scale (response scale used in PBA) from 0 to 6, Cronbach's alpha reliability for the total score of these selected five items was $\alpha$ $=.86$. The total score was highly correlated to the total score of the PBA, $r=.94$.

Next, we recoded the selected five items into the 3-point response scale created for the BPBs (see the description of the response scale development in the analysis strategy section). ${ }^{2}$ The test information curves (TIC) and related standard error (SE) functions for

\footnotetext{
${ }^{2}$ Note. Although the 3-point response scale designed for BPBs was based on content criteria, item characteristic curves for the 7-point response scale of PBA items (see Supplemental material S3) provided further support for this decision by demonstrating that the response categories selected for BPBs from PBA [i.e., response categories 7 (Every day), 6 ( A few times $a$ week), and 5 (Once a week) in S3] to reflect appearance of burnout symptoms [i.e., response options A (Daily) and B (Once or twice a week) in BPBs] were most likely to be selected by parents who were at the high end of the parental burnout continuum ( $\theta$ about $\geq$ 1.5). Category 'Every day' was most likely endorsed by respondents who had burnout levels around $\theta \geq 3$, whereas categories 'A few times a week' and 'Once a week' were most likely endorsed by respondents who showed burnout levels around $\theta \sim 2.0$ and $\theta \sim 1.5$, respectively. The response categories in the middle range of the latent parental burnout continuum (i.e., 2 , 3,4 , and 5 in S3) were somewhat overlapping. In BPBs, the categories 2, 3, and 4 were combined with the category 1 (Never) to represent the option C (More seldom/Never).
} 
BPBs (i.e., a total score of 5 items recoded into the 3-point response scale) and PBA (i.e., a total score of 23 items with 7-point response scale) items are shown in Figure 1. As indicated by maximum TIC and minimum SE (see Figure 1), the BPBs scores best assessed parental burnout at approximately $1.2 S D$ and $2.2 S D$ above the mean. Overall, BPBs scores assessed information very well from approximately the $1.2 S D$ above the mean to $2.5 S D$ above the mean, which range is well in line with the typical clinical screening range (i.e., 1.5 to $2.00 S D$ above the mean) desired for screening and identification purposes (Becker et al., 2020). The spectrum of good measurement precision for the original PBA was naturally broader (from approximately -1.5 SD below the mean to higher than 3.0 SD above the mean).

--------Insert Figure 1 about here-----

\section{Specificity and Sensitivity of the Brief Scale}

Next, the sensitivity and the specificity of the BPBs score were tested against the total score of the PBA. For the PBA, we used the established cut-off values of 86.26 (cut-off for identifying parents suffering from parental burnout) and 52.67 (cut-off for identifying parents at risk for developing clinically significant levels of parental burnout) (Brianda et al., 2020). For the BPBs, we defined three alternative cut-off values varying in their level of stringency. First, we classified the parents as burned-out or at burnout risk if they answered A (daily) or B (once or twice a week) at least in one of the five items. As a result, the cut-off value for the total score of the BPBs was 1 (i.e., the total score is obtained by summing A- (2 point), B- (1 point), and $\mathrm{C}$-answers ( 0 points); the total score ranges from 0 to 10 ; the total score with one B-answer and four C-answers is 1). This criterion was the least stringent. It promoted high sensitivity by avoiding the risk of leaving at-risk parents undetected. Second, we classified the parents as burned-out or at burnout risk if they answered A at least in one of the items or $\mathrm{B}$ at least in two of the five items. As a result, the cut-off value for the total score of the BPBs was 2 . This criterion was more stringent than the first one. Third, we classified the parents as 
burned-out or at burnout risk if they answered A at least in one of the five items and B at least in one of the four remaining items, or alternatively no A-answer but at least three B-answers. As a result, the cut-off value for the total score of the BPBs was 3. It was the most stringent.

The results of the sensitivity and specificity analyses conducted separately for the three cut-off values of 1, 2, and 3 are presented in Table 2. For the detection of burned-out parents, they showed that the sensitivity of the BPBs with the cut-off criteria of 1 and 2 compared against the PBA was $100 \%$. With the cut-off value of 3 , the sensitivity was still excellent but dropped below $100 \%$ (i.e., to $94.2 \%$ ). Specificity, in turn, was relatively high for the cut-off values of 2 and 3 but lower for the cut-off value of 1 . With the cut-off value of 1 , the frequency of false positive cases was over $20 \%$. Overall, the results suggest that the cut-off value of 2 (i.e., at least one A-answer or at least two B-answers in the BPBs) was the best value to both identify burned-out parents and limit the frequency of false positive cases.

For the detection of parents at burnout risk, the sensitivity of the BPBs with the cut-off value of 1 compared against the PBA was over $90 \%$, whereas with the values of 2 and 3, the sensitivity dropped below $80 \%$ and $60 \%$, respectively. Specificity, in turn, was relatively high for all of the three cut-off values. In particular, the frequency of false positive cases was about $15 \%$ for the cut-off value of 1 and lower than $5 \%$ for the cut-off values of 2 and 3 . Overall, the results suggest that the cut-off values of 1 (i.e., at least one A- or B-answer in the BPBs) or 2 (i.e., at least one A-answer or at least two B-answers in the BPBs) were good values to identify parents at burnout risk. The cut-off value of 1 was more sensitive to detect the risk of parental burnout than the cut-off value of 2 . However, the cut-off value of 2 displayed higher specificity than the 1-value and should be preferred when the frequency of false positives needs to be limited.

------Insert Table 2 about here----

\section{Convergent Validity of the Scores on Brief Scale}


We compared the three groups of burned-out, at burnout risk, and non-burned-out parents according to their depression symptoms, sleep disruptions, and self-esteem. The three groups were established using the three cut-off values of 1,2, and 3, so that the comparisons were computed three times. The results of the nonparametric bootstrap t-test for the depressive symptoms and self-esteem are shown in Table 3. The results showed that for the three cut-off values, both burned-out and at-risk parents demonstrated higher depressive symptoms and lower self-esteem than non-burned-out parents. According to Cohen's (1992) norms, the effect-size was large $(>.80)$ for depressive symptoms $(d=1.22,1.34$, and 1.47$)$ and medium $(>.50)$ to large for self-esteem $(d=0.79,0.93$, and 0.98$)$.

-------Insert Table 3 about here----

The results of the $\chi^{2}$-test showed that the frequency of sleep disruptions among burnedout and at-risk parents was higher than among non-burned-out parents. The difference was evident for the three cut-off values of BPBs with $\chi^{2}(1)=85.05, p<.001$ for value- $1, \chi^{2}(1)=$ 61.14, $p<.001$ for value- 2 , and $\chi^{2}(1)=41.83, p<.001$ for value-3: depending on cut-off value, $89.4 \%, 91.9 \%$ or $92.5 \%$ of burned-out or at-risk parents reported sleep disruptions, whereas the frequencies of the same sleep disruptions among the non-burned-out parents were respectively of $68.1 \%, 70.5 \%$ and $71.8 \%$.

The correlations between the level of parental burnout estimated with the BPBs and the three criteria variables (i.e., depressive symptoms, self-esteem, and sleep disruptions) are shown in Supplemental material S4. As expected, the higher the level of parental burnout, the higher the levels of depressive symptoms and sleep disruptions, and the lower the level of self-esteem. The effect sizes of the correlations were from small (sleep disruptions, $r=.279$ ) to medium (depressive symptoms, $r=.464$; self-esteem, $r=.380$ ).

\section{Study 2}


In Study 2, we went a step further. We validated the test score interpretation of BPBs developed in Study 1 by testing its sensitivity and specificity against the score of PBA. To achieve this goal, we used the BPBs and the PBA as two independent measures in an independent sample. Using the two measures in the same study is of utmost importance to ensure the validity and robustness of the test score interpretation of short measure constructed in Study 1.

\section{Method}

\section{Participants}

A total of 1,088 Finnish parents took part in a second data collection led by the IIPB Consortium. The participants (88.5\% women) had at least one child still living at home (either permanently or part-time). Most were native Finnish (96.4\%) and lived in Finland (99.3\%). The age of the participants ranged from 19 to $60(M=38.44, S D=6.81)$. The number of children ranged from 1 to $12(M=2.23, S D=1.40)$. A total of $66.9 \%$ of the participants had at least one child under school age $(<7$ year $) ; 0.6 \%(n=7)$ had only adult children (> 18 years of age). A total of $74.6 \%$ of the parents lived in a nuclear family (of which $0.5 \%$ were same-sex families), $11.2 \%$ lived in a single-parent household, and $10.2 \%$ lived in a blended family. A total of $49.2 \%$ of the participants had a university or college degree, $26.2 \%$ had a polytechnic degree, $8.3 \%$ had a technical college degree, $13.5 \%$ had a vocational school degree, and $2.8 \%$ had no vocational degree. In terms of level of education, the distribution was more representative of the Finnish population than in Study 1.

\section{Procedure}

The data collection was carried out anonymously using an online survey distributed through the social networks during the three-week Covid-19 lockdown period in Finland from April $20^{\text {th }}$ to May $13^{\text {th }}, 2020$. During the data collection period, schools were closed and parents were instructed by the state to keep children at home, maintain social distance, and 
work remotely whenever possible. The survey was presented to the participants as measuring the demands and resources the parents experienced during the lockdown.

\section{Measurements}

Parental burnout. Parental burnout was measured using both the PBA (Roskam et al., 2018) and the BPBs (developed in Study 1). In the current sample, the reliabilities (Cronbach's alpha) of the total scores of PBA and the BPBs were .97 and .84, respectively. The Spearman's non-parametric correlation between the scores of the two instruments was high, $r=.76, p<.001$.

\section{Analysis Strategy}

As in Study 1, we tested the sensitivity and the specificity of the BPBs against the PBA by the means of cross-tabulations. We used the same cut-off values as in Study 1 for the PBA (i.e., 86.26 and 52.67) and the BPBs (i.e., 1, 2, and 3).

\section{Results}

The results of the sensitivity and specificity analyses conducted separately for the three cut-off values of 1, 2, and 3 are presented in Table 2. For the detection of burned-out parents, they showed that the sensitivity of the BPBs with the three cut-off criteria ranged from $86.8 \%$ to $100 \%$. Specificity was also high for the cut-off values of 3 (the frequency of false positive cases being below 10\%) and 2 (the frequency of false positive cases being below 20\%). It was, however, lower with the cut-off value of 1 , with the frequency of false positive cases being over $30 \%$. The results suggest that the cut-off value of 2 (i.e., at least one A-answer or at least two B-answers in the BPBs) and 3 (i.e., at least one A-answer and one B-answer, or alternatively, at least three B-answers or at least two A-answers in the BPBs) were both good values to identify burned-out parents and limit the frequency of false positive cases.

For the detection of parents at burnout risk, the sensitivity of the BPBs, with the cut-off values of 1 and 2 compared against the PBA, were near $90 \%$ and $70 \%$, respectively, whereas 
the sensitivity dropped below $60 \%$ with the value of 3 . Specificity, in turn, was relatively high for the three cut-off values, the frequency of false positive cases being below $20 \%, 10 \%$, and 5\%, respectively. Overall, the results suggest that the cut-off values of 1 (i.e., at least one A- or B-answer in the BPBs) or 2 (i.e., at least one A-answer or at least two B-answers in the BPBs) were relevant values to identify parents at burnout risk. As in Study 1, the cut-off value of 1 was more sensitive in detecting parents at burnout risk than the cut-off value of 2 . The cut-off value of 2 , in turn, displayed higher specificity than the 1-value and should be preferred when the frequency of false positives needs to be limited.

\section{Study 3}

Study 3 aimed to replicate the sensitivity and specificity of the BPBs in another sample collected in a different country and move a step forward by testing the concurrent validity of the BPBs test score interpretations in relation to child neglect and parental violence.

\section{Method}

\section{Participants}

The participants were 104 French-speaking Belgian parents (93.3 \% women). The age of the participants ranged between 21 and $75(M=40.24 ; S D=9.23)$, and the number of children from 1 to $5(M=2.10, S D=0.92)$. A total of $54.8 \%$ of the participants had at least one child under school age $(<7$ year $) ; 15.4 \%(n=16)$ had only adult children $(>18$ years of age). The participants' educational level was assessed by the number of years of education they completed from the age of 6 . A total of $25.0 \%$ of the participants had a university degree at the master's or higher level (i.e., 17 years or more of education), 56.7\% had a first degree from university or college (from 12 to 15 years of education), and $24.0 \%$ were educated to the secondary level (up to 12 years of education). A total of $68.2 \%$ of the parents lived in a nuclear families (of which $2.9 \%$ were same-sex-parent families), $13.5 \%$ lived in blended families, and $11.5 \%$ lived in single-parent households. 


\section{Procedure}

Similarly to Study 2, the data were collected using an online questionnaire distributed through social networks. To avoid a self-selection bias, the survey was presented as research about parenting and its challenges and difficulties, not as research on parental burnout. The data were collected anonymously in January and February of 2019.

\section{Measurements}

Parental burnout. As in Study 2, we used both the PBA (Roskam et al., 2018) and the BPBs (French version; see Supplemental material S2). In the current sample, the reliabilities (Cronbach's alpha) of the total scores of PBA and the BPBs were .97 and .81, respectively. The correlation between the scores of the two instruments was high, $r=.82, p<.001$.

Child Neglect and Parental Violence. Child neglect and parental violence were assessed using the measure proposed by Mikolajczak et al. (2019) and Mikolajczak et al. (2020). This measure was composed of six items: one item targeting physical neglect (e.g., I don't care about my children when I know I should (meals, hygiene, etc.)), one item targeting educational neglect (e.g., I don't help my children when they really need it (on their homework, to make a decision, to resolve a conflict, etc.)), one item targeting emotional neglect (e.g., I don't comfort my children when they are sad, frightened, or distraught), one item targeting verbal violence (e.g., I say things to my children that I then regret (threats, insults, ridiculous nicknames, etc.)), one item targeting physical violence (e.g., When I get angry, I throw objects at my children or I shake my children), and one item targeting psychological violence (e.g., I tell my children that I am going to leave, and that they won't see me again if they continue to be difficult). The items are rated on an 8-point Likert scale (never or less than once a year, less than once a month, about once a month, a few times a month, about once a week, a few times a week, about once a day, a few times a day).

Cronbach's alpha reliability of the total score was 0.72 in the current sample. 


\section{Analysis Strategy}

As in Study 2, we tested the sensitivity and the specificity of the BPBs against the PBA by the means of cross-tabulations. We used the same cut-off values as in Study 1 and Study 2 for the PBA (i.e., 86.26 and 52.67) and the BPBs (i.e., 1, 2, and 3). We also tested the concurrent validity of the BPBs score interpretations. The three groups of burned-out, at-risk, and non-burned-out parents identified with the BPBs were compared to each other according to child neglect and parental violence by using a nonparametric bootstrap t-test. The correlations between the level of parental burnout estimated with the BPBs and the total score of child neglect and violence, as well as both child neglect and parental violence separately, were also calculated.

\section{Results}

\section{Sensitivity and Specificity of the BPBs}

The results of the sensitivity and specificity analyses conducted separately for the three cutoff values of 1, 2, and 3 are presented in Table 2. For the detection of burned-out parents, they showed that the sensitivity of the BPBs with the three cut-off criteria was excellent (100\%). Specificity was relatively high for the cut-off value of 3 (the frequency of false positive cases being below 15\%) but lower for the 2 -value (the frequency of false positive cases being almost 30\%) and the 1-value (over 40\%). Overall, the results showed excellent sensitivity of the BPBs. However, we found lower specificity than in Study 2. Whereas Study 2 suggested that the cut-off values of 2 and 3 were both good values to identify burned-out parents and limit the frequency of false positive cases, Study 3 suggested that a 3 -value was better than a 2-value.

For the detection of parents at burnout risk, the results showed that the sensitivity of the BPBs with the three cut-off criteria was good (> 80\%). The best sensitivity (100\%) was found for the cut-off values of 1 and 2. Specificity, in turn, was relatively high for the cut-off 
values of 2 and 3 but low for the 1-value (i.e., the frequency of false positive cases was over $30 \%$ ). Compared to Study 2, the sensitivity of the BPBs to detect parents at burnout risk was somewhat better in Study 3. On the contrary, the specificity of the BPBs was somewhat better in Study 2 than in Study 3. Overall, the results of Study 3 support our findings that the cut-off value of 2 (i.e., at least one A- or at least two B-answers in the BPBs) was relevant to identify parents at burnout risk.

\section{Concurrent Validity of the Scores on the BPBs}

We compared the three groups of burned-out, at-risk, and non-burned-out parents according to child neglect and parental violence. The three groups were established using the three cut-off values of 1,2 , and 3 , so that the comparisons were computed three times. The results of the nonparametric bootstrap t-test are displayed in Table 3. They showed that for the three cut-off values, both burned-out and at burnout risk parents demonstrated higher child neglect and parental violence than non-burned out parents. According to Cohen's (1992) norms, the effect sizes $(d=0.79, .84$, and 1.04) were medium (> .50) to large (> .80), depending on the cut-off value.

The correlations between the level of parental burnout estimated with the BPBs and the child neglect and parental violence are presented in Supplemental material S4. As expected, a higher level of parental burnout assessed with the BPBs was associated with a higher level of child neglect and parental violence. The effect size of the association was large $(r=.560)$ for the total score of child neglect and parental violence and medium for the subscale of child neglect $(r=.471)$ and subscale of parental violence $(r=.477)$.

\section{General Discussion}

The current gold standard to measure parental burnout is the PBA (Roskam et al., 2018), which has been shown to be a valid and proper method to assess parental burnout in the research context. In clinical contexts, however, more feasible and shorter tools are needed 
to identify burned-out parents and those at burnout risk. In the present research, we developed a 5-item brief screening tool (i.e., the Brief Parental Burnout Scale (BPBs)) on the basis of the 23-item PBA (PBA; Roskam et al., 2018), and we tested the validity of its test score interpretations in three successive studies. Overall, the reliability of the BPBs score and its correlation with the PBA were found to be high in three independent samples and two different cultural contexts. Compared against the PBA, the sensitivity of the BPBs score to detect both burned-out parents and parents at burnout risk was excellent. The ability of the BPBs to avoid misclassification of non-burned-out parents was mainly good. However, the specificity varied somewhat according to the cut-off values under consideration. The validity of the BPBs score interpretations was further supported by BPBs scores' associations with several external criteria.

Based on their multimethod assessment of parental burnout, Brianda et al. (2020) suggested two different cut-off values for the gold standard PBA: one for parental burnout and the other for the risk of parental burnout. Whereas the first value can be used to target interventions, the second value can be utilized to identify parents who would benefit from preventive actions. The results of the present study demonstrated, first, that the BPBs is a highly sensitive measure to identify burned-out parents. Indeed, almost all of the burned-out parents according to the PBA were classified as burned-out parents also by the BPBs. The result was replicated in two independent samples: in a sample of Finnish parents in Study 2 and in a sample of Belgian parents in Study 3.

The detection of parents displaying clinical levels of parental burnout through the test sensitivity is an important issue, as the ability of the test to detect these parents makes it possible to treat them. Because parental burnout may have detrimental outcomes, in particular child neglect and parental violence, it is crucial to identify all burned-out parents and provide them with relevant treatment. The results of the present study showed that the cut-off value of 
2 (i.e., at least one A-answer or at least two B-answers) could be considered as a relevant value for identification. Concretely, this value means that either any of the five burnout symptoms is evident daily or, alternatively, two symptoms from the five are evident once or twice a week. However, the results found for the specificity of the BPBs suggest that the cutof value of 3 would be even more optimal because the frequency of false positive cases would remain relatively low and the sensitivity would still remain over $80 \%$. Concretely, this value means that at least one of the five burnout symptoms is evident daily and that at least one another symptom is evident once or twice a week.

Second, the results showed that the BPBs test score is also a sensitive measure to detect parents who are at burnout risk. The sensitivity in this case was, however, lower than for the detection of burned-out parents. With the cut-off values of 1 and 2 , it was possible to detect 69.8-100.0\% of at-risk parents. In two separate and independent samples (Study 1 and Study 2), the cut-off value of 1 (i.e., at least one A-answer or one B-answer) was found to be the most sensitive for detecting parents at burnout risk, and in Study 3 the cut-off values of 1 and 2 were equally sensitive. In the case of the detection of parents at burnout risk, the balance between sensitivity and specificity is a very crucial issue. Indeed, when the frequency of false positive cases (i.e., parents screened to be at burnout risk although they are not at risk) is high, the cost of preventive actions increases and their quality decreases in turn. Rather than providing poor-quality preventive actions for many parents (including parents misclassified as at-risk parents), it might be more beneficial to focus on providing high-quality preventive actions for a lower number of parents who are truly at risk. The low specificity of a test can also have side effects for those screened to be at risk without a real risk. It may be a source of stress by generating thoughts or even shame about burning out in the absence of an actual threat. 
The specificity of the BPBs for the detection of parents at risk to burn out varied across the studies and the cut-off values. In Study 3, the BPBs specificity was below $70 \%$ for the cut-off value of 1 meaning that the frequency of false positives was above 30\%. In Study 2, the BPBs sensitivity, in turn, was below $60 \%$ for the cut-off value of 3 . Taken together, the findings suggest that the optimal cut-off value to identify parents at risk to burn out is 2 (i.e., at least one A- or at least two B-answers in the BPBs). With this cut-off value, it was indeed possible to identify $70 \%$ to $100 \%$ of at-risk parents and to best limit the frequency of false positive cases.

Overall, if we consider the BPBs as a screening tool to detect both burned-out parents and parents at burnout risk, the cut-off value of 2 seemed to be the most relevant value that best combined sensitivity and specificity across the two independent samples. More specifically, if high sensitivity is desired, the cut-off value of 2 should be considered as the best option. However, if a high level of specificity is desired, the cut-off value of 3 could also be considered, taking into account the health care system and the availability of the resources to provide high-quality preventive actions. If the objective is rather to mostly detect burnedout parents without missing clinical cases, lower cut-off values should be preferred to higher ones.

As expected, our results demonstrated the convergent validity of the scores on BPBs. Previous literature has shown that parental burnout is associated with several correlates, such as depressive symptoms (Aunola et al., 2020; Mikolajczak et al., 2019; Van Bakel et al., 2018), low self-esteem (Aunola et al., 2020), and sleeping disruptions (Aunola et al., 2020; Lindström et al., 2011; Mikolajczak et al., 2018). Parental burnout has also been shown to predict specific outcomes, namely child neglect and parental violence (Mikolajczak et al., 2018; Mikolajczak et al., 2019). In line with the literature, we found the expected associations between parental burnout measured with the 5-item combination selected for the BPBs and 
these variables. In particular, Study 1 demonstrated that with the three cut-off values of the BPBs, burned-out parents and parents at burnout risk displayed a significantly higher level of depressive symptoms and a lower level of self-esteem than non-burned-out parents. They were also more likely to report sleeping disruptions. Furthermore, Study 3 demonstrated that burned-out parents and parents at burnout risk reported a higher level of child neglect and parental violence than non-burned-out parents. Also, as expected, the effect sizes of the correlations between the BPBs and the criteria variables were found to be consistent with those found in the previous literature, with the effect sizes varying from small (for sleep disruptions) or medium (depressive symptoms, self-esteem) to large (child neglect and parental violence).

In spite of the convincing results and the three complementary studies that were carried out, our research is not without limitations. First, the samples of the three studies were not random samples, and we therefore cannot rule out selection biases among the parents who participated. Second, fathers were under-represented in all three studies, and lower-educated parents were also under-represented, particularly in Study 1. The results should be replicated using gender-balanced samples as well as samples with diverse sociodemographic backgrounds. Third, in Study 3 the proportion of participants whose children were already adults was relatively high (about 15\%) and the sample size was also smaller (being reflected as wider confidence intervals in statistical testing) than in the other two studies. These factors may have affected the results of Study 3 and, for example, partly explain the lower specificity of BPBs score in Study 3. Fourth, the validity of the BPBs score interpretations was tested in two different countries, i.e., Finland and Belgium. Both countries are considered Western cultures with individualistic values and relatively well-developed health care systems. Future studies are needed to validate the test score interpretations of the BPBs in other countries, cultural contexts, and among different subpopulations. It is possible, for example, that due to 
cultural and societal effects, the sensitivity and the specificity of the BPBs varies and that the optimal cut-off values would be culture specific rather than universal. Finally, as BPBs was developed to be as a screening tool, its' measurement precision does not extend across the full spectrum of the latent parental burnout trait. Thus, although the BPBs score is able to differentiate between respondents across the typical clinical range, it does not differentiate between respondents at the lower half of the latent trait continuum (i.e., there are not enough response options for the lower half of the latent trait continuum). As a screening tool, the BPBs is developed for the categorizations and is not designed to be used for continuous scoring. Therefore, the BPBs score should not be used to assess the level of parental burnout symptoms among parents with low levels of parental burnout. Despite these limitations, the results of the present study suggest that the BPBs score has strong psychometric properties as a screening tool. Furthermore, because of these properties and its user-friendliness and feasibility, the BPBs can be considered a suitable option to be used in health care settings as a screening tool for parental burnout or the risk of it. This will enable the development of interventions for burned-out parents and/or preventive steps for those parents who are at burnout risk. Since parental burnout is a serious condition that can affect all kinds of parents, it is essential that this kind of screening tool be available for use as a standard, low-threshold procedure nationwide. The BPBs could be successfully used, for example, in local child health centers by nurses, doctors, or psychologists, or as part of social services for families. 


\section{References}

Arikan, G., Üstündağ-Budak, A. M., Akün, E., Mikolajczak, M., \& Roskam, I. (2020). Validation of the Turkish version of the Parental Burnout Assessment (PBA). New Directions for Child and Adolescent Development, 1-18. doi: 10.1002/cad.20375

Aunola, K., Sorkkila, M., \& Tolvanen, A. (2020). Validity of the Finnish version of the Parental Burnout Assessment (PBA). Scandinavian Journal of Psychology, 61, 714722. doi: $10.1111 /$ sjop. 12654

Baker, F. (2001). Basics of item response theory. Retrieved from http://echo.edres.org:8080/irt/baker

Beck, A. T., Ward, C. H., Mendelsohn, M., Mock, L., \& Erlaugh, J. (1961). An inventory for measuring depression. Archives of General Psychiatry, 4, 561-571.

Becker, S. P., Mossing, K. W., Zoromski, A. K., Vaughn, A. V., Epstein, J. N., Tamm, L., \& Burns, G. L. (2020). Assessing sluggish cognitive tempo and ADHD inattention in elementary students: Empirical differentiation, invariance across sex and Grade, and measurement precision. Psychological Assessment, 32, 1047-1056. doi: 10.1037/pas0000946

Bianchi, R., Truchot, D., Laurent, E., Brisson, R., \& Schonfeld, I. S. (2014). Is burnout solely job-related? A critical comment. Scandinavian Journal of Psychology, 55, 357-361. doi: 10.1111/sjop.12119

Blanchard, A-L., Roskam, I., Mikolajczak, M., \& Heeren, A. (2020). A network approach to parental burnout. PsyArXiV. doi: 10.31234/osf.io/swqfz

Brianda, M. E., Mikolajczak, M., Bader, M., Bon, S., Déprez, A., Favez, N., Holstein, L., Le Vigouroux, S., Lebert-Charron, A., Sánchez-Rodríguez, R., Séjourné, N., Wendland, J., \& Roskam, I. (2020). Optimizing screening for parental burnout: A multi-informant 
and multi-method approach to determine cut-offs for the Parental Burnout Inventory (PBI) and the Parental Burnout Assessment (PBA). Pre-registrated manuscript draft.

Chen, H., Wang, W., Wang, S., Li, Y., Liu, X., \& Youngxin, L. (2020). Validation of a Chinese version of the Parental Burnout Assessment (PBA). Frontiers of Psychology, 11,321 .

Cohen, J. (1992). A power primer. Psychological Bulletin, 112, 155-159.

Crnic, K., \& Low, C. (2002). Everyday stresses and parenting. In M. H. Bornstein (Ed.), Handbook of parenting: Practical issues in parenting (pp. 243-267). Lawrence Erlbaum Associates Publishers.

Furutani, K., Kawamoto, T., Alimardani, M., \& Nakashima, K. (2020). Exhausted parents in Japan: Preliminary validation of the Japanese version of the Parental Burnout Assessment. New Directions for Child and Adolescent Development, 1-17. doi: 10.1002/cad.20371

Hansotte, L., Nguyen, N., Roskam, I., Stinglhamber, F., \& Mikolajczak, M. (2021). Are all burned out parents neglectful and violent? A latent profile analysis. Journal of Child and Family Studies, 30, 158-168. doi: 10.1007/s10826-020-01850-x.

Lindström, C., Åman, J., \& Norberg, A.L. (2011). Parental burnout in relation to sociodemographic, psychosocial and personality factors as well as disease duration and glycaemic control in children with Type 1 diabetes mellitus. Acta Paediatrica, 100, $1011-1017$.

Maslach, C., Jackson, S. E., \& Leiter, M. P. (1997). Maslach, Burnout Inventory, 3rd Edition. In C. P. Zalaquett \& R. J. Wood (Eds.), Evaluating stress: A book of resources (pp. 191-218). The Scarecrow Press.

Maslach, C., Schaufeli, W. B., \& Leiter, M. P. (2001). Job burnout. Annual Review of Psychology, 52, 397-422. 
Matias, M., Aguiar, J., César, F., Braz, C. A., Barham, E. J., Leme, V., Elias, L., Gaspar, M. F., Mikolajczak, M., Roskam, I., \& Fontaine, A. M. (2020). The Brazilian-Portuguese version of the Parental Burnout Assessment: Transcultural adaptation and initial validity evidence. New Directions for Child and Adolescent Development. doi:10.1002/cad.20374

Mikolajczak, M., \& Roskam, I. (2018). A theoretical and clinical framework for parental burnout: The balance between risks and resources (BR2). Frontiers of Psychology, 9, 886. doi: 10.3389/fpsyg.2018.00886

Mikolajczak, M., Brianda, M.E., Avalosse, H., \& Roskam, I. (2018). Consequences of parental burnout: Its specific effect on child neglect and violence. Child Abuse and Neglect, 80, 134-145.

Mikolajczak, M., Gross, J.J., \& Roskam, I. (2019). Parental burnout: What is it, and why does it matter? Clinical Psychological Science, 7, 1319-1329. doi: $10.1177 / 2167702619858430$

Mikolajczak, M., Gross, J., Stinglhamber, F., Lindahl Norberg, A., \& Roskam, I. (2020). Is parental burnout distinct from job burnout and depressive symptomatology? Clinical Psychological Science, 8, 673-689.

Mousavi, S.F., Mikolajczak, M., \& Roskam, I. (2020). Parental burnout in Iran: Psychometric properties of the Persian (Farsi) version of the Parental Burnout Assessment (PBA). New Directions for Child and Adolescent Development. doi:10.1002/cad.20369

Muthén, L. K., \& Muthén, B. O. (1998-2019). Mplus users' guide (8th ed.). Muthén and Muthén.

Olino, T. M.,. Yu, L., Klein, D. N., Rohde, P., Seeley, J. R., Pilkonis, P. A., \& Lewinsohn, P. M. (2012). Measuring depression using item response theory: An examination of three 
measures of depressive symptomatology. International Journal of Methods in Psychiatric Research, 21, 76-85. doi: 10.1002/mpr.1348

Parker, P. D., \& Salmela-Aro, K. (2011). Developmental processes in school burnout: A comparison of major developmental models. Learning and Individual Differences, 21, 244-248. doi:10.1016/j.lindif.2011.01.005

Rosenberg, M. (1979). Conceiving the self. Basic Books.

Roskam, I., Aguiar, J., Akgun, E., Arikan, G., Artavia, M., Avalosse, H., Aunola, K., Bader, M., Bahati, C., Barham, E. J., Besson, E., Beyers, W., Boujut, E., Brianda, M. E., Brytek-Matera, A., Carbonneau, N., César, F., Chen, B.-B., Dorard, G., . . . Mikolajczak, M. (2021). Parental burnout around the globe: A 42-country study. Affective Science, 2, 58-79. doi: 10.1007/s42761-020-00028-4

Roskam, I., Brianda, M.-E., \& Mikolajczak, M. (2018). A step forward in the conceptualization and measurement of parental burnout: The Parental Burnout Assessment (PBA). Frontiers of Psychology, 9, doi: 10.3389/fpsyg.2018.00758

Roskam, I., \& Mikolajczak, M. (under review). The slippery slope of parental exhaustion: A stage model of parental burnout.

Roskam, I., Raes, M.-E., \& Mikolajczak, M. (2017). Exhausted parents: Development and preliminary validation of the Parental Burnout Inventory. Frontiers of Psychology, 8, https://doi.org/10.3389/fpsyg.2017.00163

Stănculescu, E., Roskam, I., Mikolajczak, M., Muntean, A., \& Gurza, A. (2020). Parental burnout in Romania: Validity of the Romanian version of the parental burnout assessment (PBA-RO). New Directions for Child and Adolescent Development. doi:10.1002/cad.20384 
Szczygieł, D., Sekulowicz, M., Kwiatkowski, P., Roskam, I., \& Mikolajczak, M. (2020). Validation of the Polish version of the Parental Burnout Assessment (PBA). New Directions for Child and Adolescent Development, 1-22. doi: 10.1002/cad.20385

Taris, T. W., Le Blanc, P. M., Schaufeli, W. B., \& Schreurs, P. J. G. (2005). Are there causal relationships between the dimensions of the Maslach Burnout Inventory? A review and two longitudinal tests. Work \& Stress, 19, 238-255. doi: 10.1080/02678370500270453

Van Bakel, H. J. A., Van Engen, M. L., \& Peters, P. (2018). Validity of the Parental Burnout Inventory among Dutch employees. Frontiers in Psychology, 9, 697. doi: 10.3389/fpsyg.2018.00697

Versi, E. (1992). “Gold standard” is an appropriate term. British Medical Journal, 305 (6846): 187. doi: 10.1136/bmj.305.6846.187-b 


\section{Table 1}

Distributions of the Parental Burnout Items (7-Category Scale*), and Results of Item Response Theory Graded Response Model Analyses (the

Final Selected Items in Bold)

\begin{tabular}{|c|c|c|c|c|c|c|c|c|c|c|c|c|c|c|c|}
\hline & \multicolumn{7}{|c|}{ Response frequencies (\%) } & \multicolumn{7}{|c|}{ Item parameter estimates } & \multirow{2}{*}{$\begin{array}{l}\text { Relative } \\
\text { difficulty } \\
\text { (severity) }\end{array}$} \\
\hline & 0 & 1 & 2 & 3 & 4 & 5 & 6 & $\alpha$ & $\mathrm{b}_{1}$ & $\mathrm{~b}_{2}$ & $\mathrm{~b}_{3}$ & $\mathrm{~b}_{4}$ & $\mathrm{~b}_{5}$ & $\mathrm{~b}_{6}$ & \\
\hline EX3 & 34.0 & 26.2 & 11.8 & 12.1 & 5.0 & 7.5 & 3.4 & 2.71 & -0.46 & 0.27 & 0.65 & 1.16 & 1.48 & 2.27 & Low \\
\hline CO6 & 38.3 & 31.4 & 11.5 & 8.5 & 3.6 & 5.8 & 0.9 & 2.40 & -0.36 & 0.56 & 1.03 & 1.54 & 1.87 & 3.10 & Medium \\
\hline EX1 & 34.9 & 27.3 & 12.5 & 10.2 & 4.7 & 6.8 & 3.6 & 3.29 & -0.40 & 0.31 & 0.71 & 1.14 & 1.42 & 2.12 & Low \\
\hline $\mathrm{EX}^{1}$ & 52.0 & 29.1 & 7.4 & 6.3 & 1.9 & 2.7 & 0.7 & 2.40 & 0.04 & 0.90 & 1.44 & 2.04 & 2.33 & 3.21 & High \\
\hline $\mathrm{CO} 1$ & 41.7 & 28.7 & 9.5 & 7.7 & 3.9 & 5.6 & 3.0 & 2.09 & -0.28 & 0.60 & 0.99 & 1.45 & 1.77 & 2.53 & Medium \\
\hline $\mathrm{FU} 1^{1}$ & 66.8 & 17.3 & 6.1 & 5.4 & 1.3 & 2.6 & 0.5 & 2.72 & 0.47 & 1.12 & 1.51 & 2.07 & 2.30 & 3.23 & High \\
\hline FU3 & 49.3 & 26.4 & 10.2 & 5.2 & 3.4 & 3.8 & 1.7 & 2.59 & -0.02 & 0.79 & 1.26 & 1.64 & 1.99 & 2.69 & Medium \\
\hline $\mathbf{E X 8}^{3}$ & 18.3 & 26.2 & 19.9 & 13.6 & 6.6 & 10.7 & 4.6 & 2.17 & -1.13 & -0.20 & 0.43 & 0.96 & 1.30 & 2.23 & Low \\
\hline $\mathbf{E X} 2^{2}$ & 33.5 & 31.0 & 12.1 & 10.1 & 4.6 & 6.3 & 2.4 & 3.69 & -0.43 & 0.37 & 0.76 & 1.23 & 1.53 & 2.29 & Medium \\
\hline EX4 & 29.7 & 29.3 & 13.4 & 10.6 & 4.5 & 7.9 & 4.5 & 2.61 & -0.60 & 0.26 & 0.69 & 1.15 & 1.41 & 2.11 & Low \\
\hline $\mathrm{FU}^{1}$ & 33.6 & 28.2 & 15.1 & 9.4 & 5.2 & 7.1 & 1.3 & 2.44 & -0.49 & 0.33 & 0.86 & 1.32 & 1.70 & 2.87 & Medium \\
\hline
\end{tabular}




\begin{tabular}{|c|c|c|c|c|c|c|c|c|c|c|c|c|c|c|c|}
\hline $\mathrm{FU}^{1}{ }^{1}$ & 34.2 & 32.7 & 12.8 & 9.3 & 3.7 & 5.6 & 1.7 & 2.64 & -0.46 & 0.46 & 0.94 & 1.45 & 1.77 & 2.67 & Medium \\
\hline $\mathrm{CO} 2^{1}$ & 45.7 & 26.1 & 8.7 & 8.6 & 3.8 & 4.8 & 2.3 & 1.97 & -0.17 & 0.69 & 1.08 & 1.63 & 1.99 & 2.80 & Medium \\
\hline ED1 & 42.3 & 25.8 & 13.5 & 8.6 & 3.6 & 3.9 & 2.3 & 1.43 & -0.32 & 0.65 & 1.34 & 2.01 & 2.45 & 3.30 & High \\
\hline EX7 & 33.4 & 24.7 & 12.2 & 10.8 & 6.3 & 8.5 & 4.1 & 2.86 & -0.47 & 0.23 & 0.61 & 1.04 & 1.37 & 2.12 & Low \\
\hline $\mathrm{FU}{ }^{1}$ & 72.4 & 15.8 & 4.9 & 3.5 & 1.2 & 1.6 & 0.5 & 2.87 & 0.64 & 1.32 & 1.73 & 2.21 & 2.48 & 3.25 & High \\
\hline $\mathrm{CO}^{1}$ & 41.1 & 28.4 & 11.0 & 8.4 & 3.3 & 4.5 & 3.4 & 2.64 & -0.26 & 0.55 & 0.96 & 1.41 & 1.68 & 2.27 & Low \\
\hline $\mathrm{CO} 4$ & 27.8 & 31.9 & 14.0 & 11.0 & 4.8 & 5.9 & 4.7 & 2.87 & -0.64 & 0.26 & 0.69 & 1.16 & 1.47 & 2.04 & Low \\
\hline $\mathrm{CO} 5$ & 50.6 & 23.1 & 9.3 & 7.9 & 2.6 & 4.2 & 2.3 & 2.65 & -0.01 & 0.66 & 1.06 & 1.56 & 1.82 & 2.50 & Medium \\
\hline $\mathbf{E D 3}^{3}$ & 70.3 & 15.1 & 6.6 & 3.6 & 1.7 & 1.9 & 0.8 & 1.63 & 0.71 & 1.44 & 1.97 & 2.47 & 2.86 & 3.75 & High \\
\hline EX5 & 36.8 & 27.9 & 13.1 & 9.3 & 4.5 & 5.2 & 3.1 & 2.78 & -0.38 & 0.40 & 0.86 & 1.32 & 1.63 & 2.27 & Medium \\
\hline ED2 & 32.0 & 27.5 & 15.6 & 12.2 & 5.0 & 5.8 & 1.8 & 2.22 & -0.58 & 0.27 & 0.82 & 1.44 & 1.84 & 2.78 & Medium \\
\hline $\mathrm{EX}^{1}$ & 37.2 & 28.5 & 11.4 & 9.3 & 4.5 & 4.9 & 4.3 & 2.98 & -0.36 & 0.43 & 0.82 & 1.25 & 1.55 & 2.06 & Low \\
\hline
\end{tabular}

Note 1. * Response categories were as follows: $0=$ Never, $1=\mathrm{A}$ few times a year, $2=$ Once a month, $3=\mathrm{A}$ few times a month, $4=$ Once a week, $5=$ A few times a week, $6=$ Every day.

Note2. $\alpha=$ item discrimination parameter; $\mathrm{b}_{1}-\mathrm{b}_{6}=$ item difficulty (severity) parameters for different thresholds; EX $=$ Exhaustion in one's parental role, $\mathrm{CO}=$ Contrast with previous parental self, $\mathrm{FU}=$ Feelings of being fed up with parenting, ED = Emotional distancing from one's child(ren)

Note $3 .{ }^{1}$ Not appropriate from content point of view; ${ }^{2}$ appropriate because the best item discrimination and appropriate content (medium difficult level); ${ }^{3}$ appropriate because represents extremity (low or high item difficulty) and appropriate content. 


\section{Table 2}

Sensitivity and Specificity of BPBs ${ }^{1}$ Compared Against the Gold Standard PBA in Study 1 (Finnish data; $\left.n=1,725\right)$, Study 2 (Finnish data; $n=$ 1,088), and Study 3 (Belgian data; $n=104$ ): Results of Cross-tabulation Using Different Cut-off Values

\begin{tabular}{|c|c|c|c|c|c|c|c|c|c|c|}
\hline \multirow[t]{2}{*}{$\begin{array}{l}\text { Cut-off value } \\
\text { PBA }\end{array}$} & \multirow[t]{2}{*}{$\begin{array}{l}\text { Cut-off value } \\
\text { BPBs }^{1}\end{array}$} & \multicolumn{3}{|c|}{$\begin{array}{l}\text { Sensitivity } \\
\text { (\% of true positive) }\end{array}$} & \multicolumn{3}{|c|}{$\begin{array}{l}\text { Specificity } \\
\%\end{array}$} & \multicolumn{3}{|c|}{$\begin{array}{l}\text { 100-specificity } \\
\text { (\% of false positive) }\end{array}$} \\
\hline & & $\begin{array}{l}\text { Study } 1 \\
\text { (PBA/ } \\
5 \text { items) }\end{array}$ & $\begin{array}{l}\text { Study } 2 \\
\text { (BPBs) }\end{array}$ & $\begin{array}{l}\text { Study3 } \\
\text { (BPBs) }\end{array}$ & $\begin{array}{l}\text { Study } 1 \\
\text { (PBA/ } \\
5 \text { items) }\end{array}$ & $\begin{array}{l}\text { Study } 2 \\
\text { (BPBs) }\end{array}$ & $\begin{array}{l}\text { Study3 } \\
\text { (BPBs) }\end{array}$ & $\begin{array}{l}\text { Study } 1 \\
\text { (PBA/ } \\
5 \text { items) }\end{array}$ & $\begin{array}{l}\text { Study } 2 \\
\text { (BPBs) }\end{array}$ & $\begin{array}{l}\text { Study3 } \\
\text { (BPBs) }\end{array}$ \\
\hline \multirow[t]{3}{*}{$86.26^{2}$} & 1 & 100.0 & 100.0 & 100.0 & 75.0 & 65.9 & 58.2 & 25.0 & 34.1 & 41.8 \\
\hline & 2 & 100.0 & 93.4 & 100.0 & 87.2 & 83.3 & 73.5 & 12.8 & 16.7 & 26.5 \\
\hline & 3 & 94.2 & 86.8 & 100.0 & 92.8 & 92.4 & 85.7 & 7.2 & 7.6 & 14.3 \\
\hline \multirow[t]{3}{*}{$52.67^{3}$} & 1 & 90.2 & 88.8 & 100.0 & 85.2 & 80.5 & 63.3 & 14.8 & 19.5 & 36.7 \\
\hline & 2 & 73.2 & 69.8 & 100.0 & 95.4 & 94.8 & 80.0 & 4.6 & 5.2 & 20.0 \\
\hline & 3 & 58.6 & 52.1 & 85.7 & 98.8 & 98.9 & 91.1 & 1.2 & 1.1 & 8.9 \\
\hline
\end{tabular}

Note. ${ }^{1}$ In Study 1, the data collection included only the PBA and, therefore, the BPBs in Study 1 refers to the five items statistically selected from the PBA and recoded on the developed scaling system (range 0-2). In Studies 2 and 3, the data collection included both the PBA and the BPBs as separate scales. ${ }^{2} \mathrm{~A}$ total of $6.0 \%$ of the parents in Study 1, 14.0\% of the parents in Study 2, and 5.8\% of the parents in Study 3 scored higher or equal than the cut-off score 86.26 (i.e., cut-off for parental burnout) in the PBA. ${ }^{3}$ A total of $19.5 \%$ of the parents in Study 1 , $34.4 \%$ in Study 2, and $13.5 \%$ in Study 3 scored higher or equal than the cut-off score 52.67 (i.e., cut-off for the risk of parental burnout) in the gold standard PBA. 


\section{Table 3}

Differences Between Burned-Out Parents or Parents at Burnout Risk According to the BPBs ${ }^{l}$ and Non-Burned-Out Parents in Depressive Symptoms

(Study 1), Self-Esteem (Study 1), and Child Neglect/Parental Violence (Study 3)(Results of the Nonparametric Bootstrapped t-test)

\begin{tabular}{|c|c|c|c|c|c|c|c|c|c|c|}
\hline \multicolumn{4}{|c|}{ Depressive Symptoms (Study 1) } & \multicolumn{3}{|c|}{ Self-Esteem (Study 1) } & \multicolumn{4}{|c|}{ Child Neglect/Parental Violence (Study 3) } \\
\hline$n$ & $M(S D)$ & $t$ & $95 \% C I$ & $M(S D)$ & $t$ & $95 \% C I$ & $n$ & $M(S D)$ & $t$ & $95 \% C I$ \\
\hline 505 & $3.63(0.72)$ & $-23.69^{a}$ & $3.57-3.69$ & $3.51(0.74)$ & $14.29^{\mathrm{a}}$ & $3.45-3.58$ & 47 & $11.62(5.14)$ & $-3.86^{\mathrm{b}}$ & $10.27-13.20$ \\
\hline 1214 & $2.67(0.85)$ & & $2.62-2.72$ & $4.05(0.63)$ & & $4.02-4.08$ & 57 & $8.51(2.21)$ & & $8.00-9.10$ \\
\hline
\end{tabular}

Cut-off value 1

Burned-out/At-risk group

Non-Burned-out

\section{Cut-off value 2}

Burned-out/At-risk group

Non-Burned-out

\section{Cut-off value 3}

Burned-out/At-risk group

Non-Burned-out
$12142.67(0.85)$

$2.62-2.72 \quad 4.05(0.63)$

$4.02-4.08$
$3073.81(0.67)$

$14122.77(0.87)$

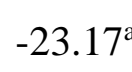

(5)

$14.14^{\mathrm{a}} 3.27-3.45$

$3.97-4.04$
$32 \quad 12.50(5.90) \quad-3.48^{\mathrm{b}} \quad 10.67-14.54$ $72 \quad 8.76(2.22) \quad 8.27-9.32$

$2113.95(0.66) \quad-23.94^{\mathrm{a}} \quad 3.86-4.03 \quad 3.28(0.76) \quad 12.71^{\mathrm{a}} 3.18-3.38$ $15082.81(0.88)$

Note. ${ }^{1}$ In study 1 , the BPBs refers to the five items statistically selected from the PBA and recoded on the developed response scale (0-2). In Study 3, the data collection included both the PBA and the BPBs as separate scales; ${ }^{\mathrm{a}} p<.001,{ }^{\mathrm{b}} p<.01,{ }^{\mathrm{c}} p<.05$; CI $=$ Bootstrapped $95 \%$ confidence interval. 


\section{Figure 1}

Test Information Curves (TIC) for the 23-Item Parental Burnout Assessment (PBA) and 5Item Brief Parental Burnout Scale (BPBs) (the Top Panel of the Figure) and Standard Error Functions for PBA and BPBs Items (the Bottom Panel of the Figure). A Total Information of 10 Correspond to a Reliability Coefficient of .90 and Values Around 5 Correspond to a Reliability of .80. Note. In the Study 1, BPBs refers to the selected 5 items of PBA that were re-coded on the 3-point response scale.
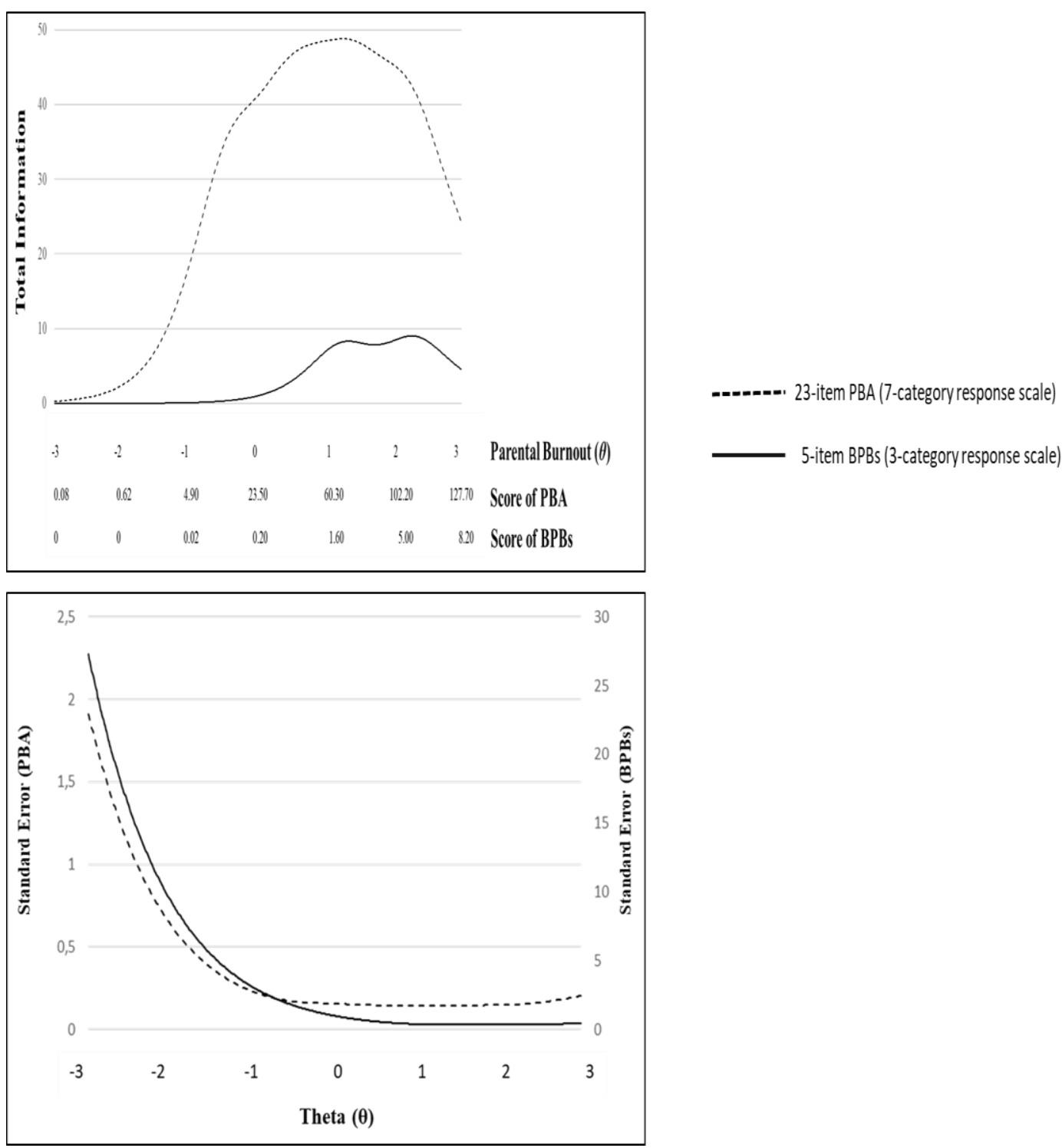
APPENDIX A. Brief Parental Burnout Scale (BPBs) [Note 1. In the BPBs, the items are presented in the following order: EX3, EX2, EX8, ED3, FU3. Note 2. Due to feedback received from parents and nurses in the practical piloting of BPBs, the third item EX8 was extended from the original EX8 by including an additional clarification in parentheses.].

\section{EVEN PARENTS CAN BURN OUT}

Exhaustion is a feeling that can be experienced by any parent. On the one hand, you can love your children deeply and be grateful for them, and, on the other hand, experience exhaustion in your role as a parent. These feelings do not rule each other out. For the sake of your own well-being, it is important to recognize these feelings as early as possible. Help is available. When you think about your role as a parent, how often do you feel the following way? Please circle the option that best matches your experience from each row below.

Daily Once or twice More a week seldom/

Never

I'm so tired out by my role as a parent that sleeping doesn't seem like enough.

A

B

C

I have the sense that I'm really worn out as a parent.

A

B

C

I have the impression that I'm looking after my child(ren) on autopilot

A

B

C

(I do what I'm supposed to do for my child(ren), but nothing more).

I'm no longer able to show my child(ren) how much I love them.

A

B

C

I feel like I can't take any more as a parent.

A 


\section{Supplemental material S1:}

\section{Original version of the Brief Parental Burnout scale (BPBs) in Finnish}

(Note. In the BPBs, the items are presented in the following order: EX3, EX2, EX8,

ED3, FU3).

VAU - Vanhemmuuden uupumusseula

\section{VANHEMPANAKIN VOI UUPUA}

Uupumus on tunne, jota kuka tahansa vanhempi saattaa tuntea. Voit yhtäältä rakastaa lapsiasi syvästi ja iloita heistä, ja toisaalta kokea uupumusta vanhemman roolissa. Nämä tunteet eivät sulje toisiaan pois. Oman jaksamisesi ja hyvinvointisi vuoksi on tärkeää tunnistaa nämä tunteet ajoissa. Tukea on tarvittaessa saatavilla. Kun mietit omaa rooliasi vanhempana, kuinka usein koet seuraavilla tavoilla? Ympäröi kokemustasi parhaiten vastaava vaihtoehto kultakin riviltä. pari viikossa Ei koskaan

Olen niin väsynyt rooliini vanhempana, ettei edes nukkuminen auta.

A

B

C

Tunnen olevani aivan lopen uupunut vanhempana.

A

B

C

Tuntuu kuin huolehtisin lapsestani/lapsistani automaattiohjauksella (teen vain välttämättömät asiat mutta en enempää).

En enää pysty näyttämään lapselleni/lapsilleni, kuinka paljon rakastan häntä/heitä.

A

B

C

Minusta tuntuu, etten jaksa enempää vanhempana.

A

B

C 


\section{Supplemental material S2:}

Translated version of the Brief Parental Burnout scale (BPBs) in French

(Note. In the BPBs, the items are presented in the following order: EX3, EX2, EX8, ED3, FU3)

J'ai l'impression de m'occuper de mes enfants en pilote automatique

A $\quad$ B $\quad$ C

Je n'arrive plus à montrer à mes enfants combien je les aime

A

B

$\mathrm{C}$

J'ai le sentiment que je n'en peux vraiment plus en tant que parent 


\section{Supplemental material S4}

Correlations of the BPBs ${ }^{1}$ and the PBA With Depressive Symptoms (Study 1), Self-

Esteem (Study 1), Sleep Disruptions (Study 1), and Child Neglect and Parental Violence

(Study 3)

PBA $\quad$ BPBs $^{1}$

Study $1(n=1725)$

Depressive symptoms

$.632 \quad .464$

Self-esteem

$-.493 \quad-.380$

Sleep disruptions (total score of the three items)

$.354 \quad .279$

item 1

$.326 \quad .239$

item2

$.212 \quad .169$

item3

$.219 \quad .194$

Study $3(n=104)$

Child neglect and Parental violence (total score of the six items)

$.659 \quad .560$

child neglect (three items)

$.584 \quad .471$

parental violence (three items)

$.535 \quad .477$

Note. ${ }^{1}$ In Study 1, the BPBs refers to the five items statistically selected from the PBA and recoded on the developed response scale (0-2). In Study 3, the data collection included both the PBA and the BPBs as independent scales.

Note 2. All correlations are statistically significant at $p<.001$.

Note 3. Item 1 of sleep disruptions is "Do you feel that you get enough sleep?", item 2 is "Can you easily fall asleep in the evenings?", and item 3 is "Do you often wake up at night and cannot get back to sleep again?" (Reversed item). Parents answered for the items either "yes" (value 1) or "no" (value 2). 


\section{Supplemental material S3: The item characteristics and information curves} of PBA items (based on data of 1, 725 parents).

Note 1. In 23-item PBA, items are rated using 7-point response scale from 0 (Never) to 6 (Every day). In the Figures of Supplemental material S3, Category 1 refers to the response option 0 (Never) of PBA, Category 2 refers to the response option 1 (Afew times a year), Category 3 refers to the response option 2 (Once a month), Category 4 refers to the response option 3 (A few times a month),

Category 5 refers to the response option 4 (Once a week), Category 6 refers to the response option 5 (Afew times a week), and Category 7 refers to the response option 6 (Every day).

Note 2. $\mathrm{Ex}=$ exhaustion in one's parental role; $\mathrm{Co}=$ contrast with the previous parental self; $\mathrm{Fu}=$ feelings of being fed up as a parent; $\mathrm{Ed}=$ emotional distancing from one's children.

Note 3. The five items selected for the Brief Parent Burnout scale (BPBs) are outlined. 
BRIEF PARENTAL BURNOUT SCALE

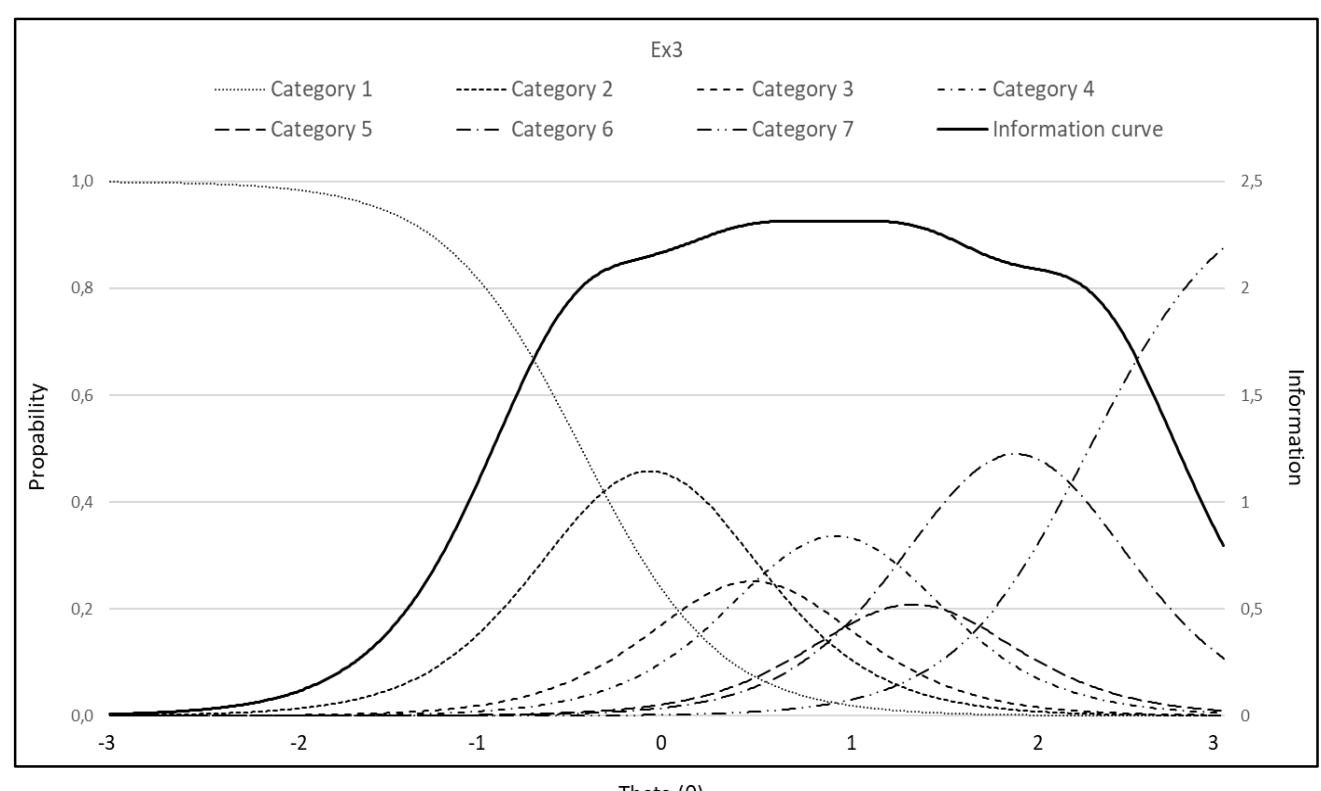

Ex1

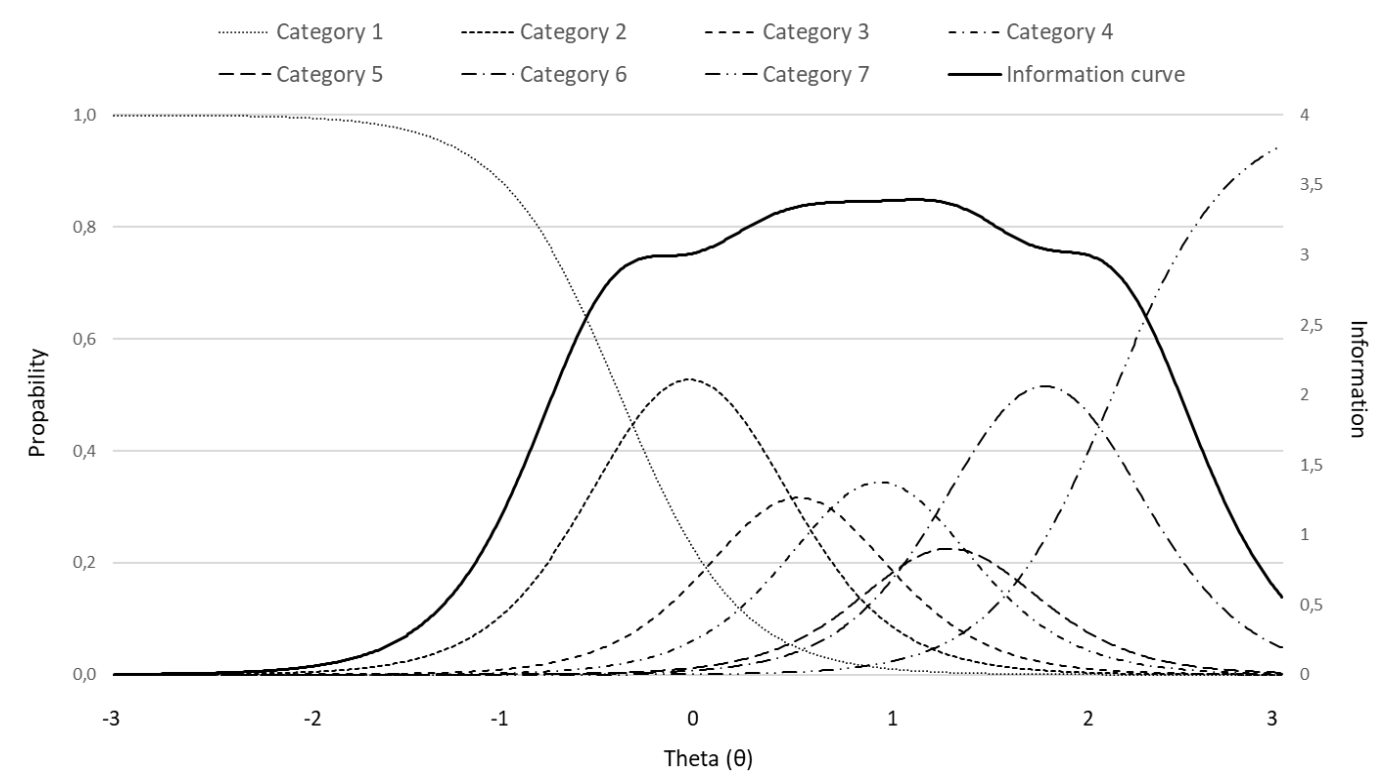

45
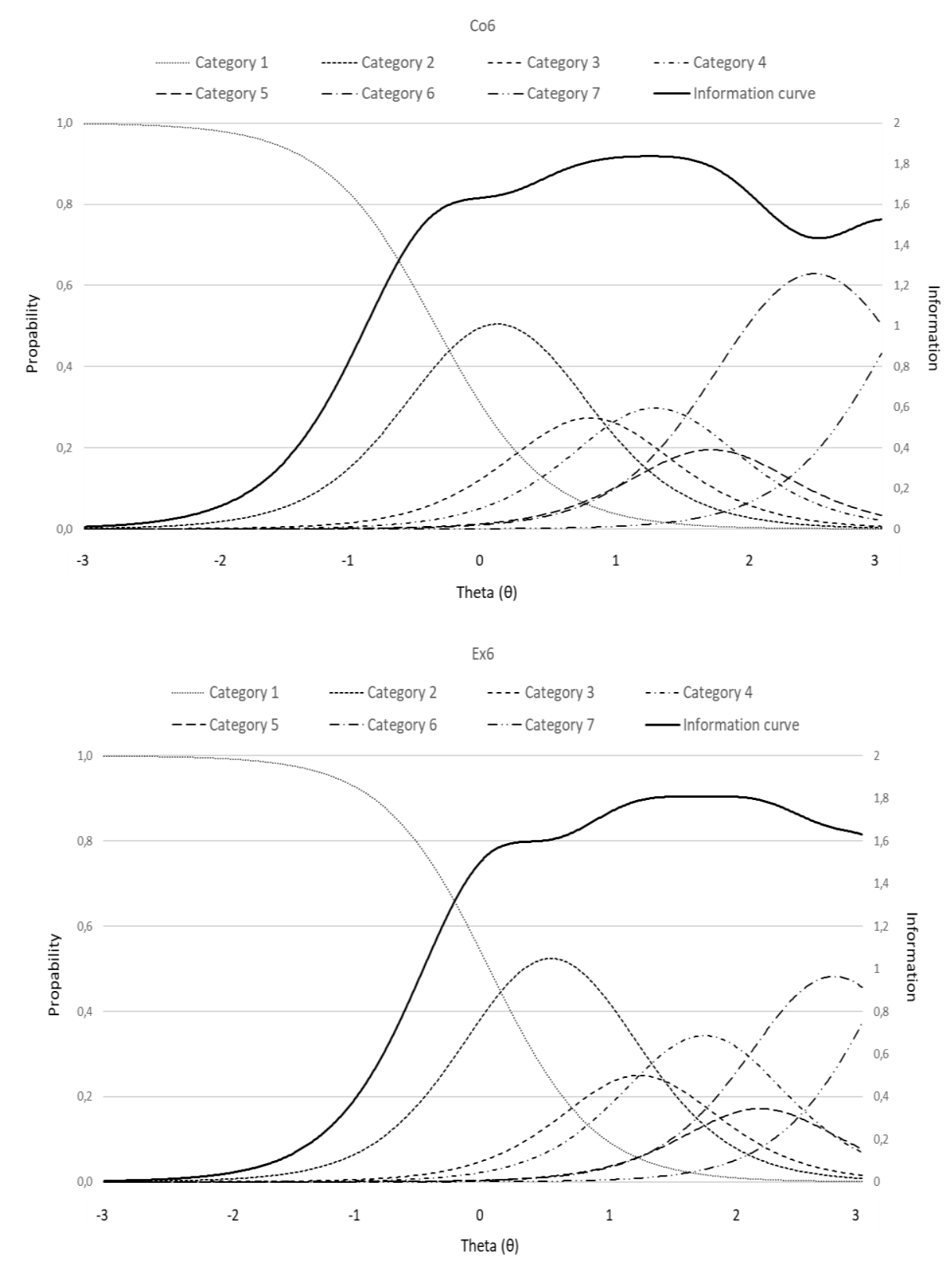


\section{BRIEF PARENTAL BURNOUT TCALE}
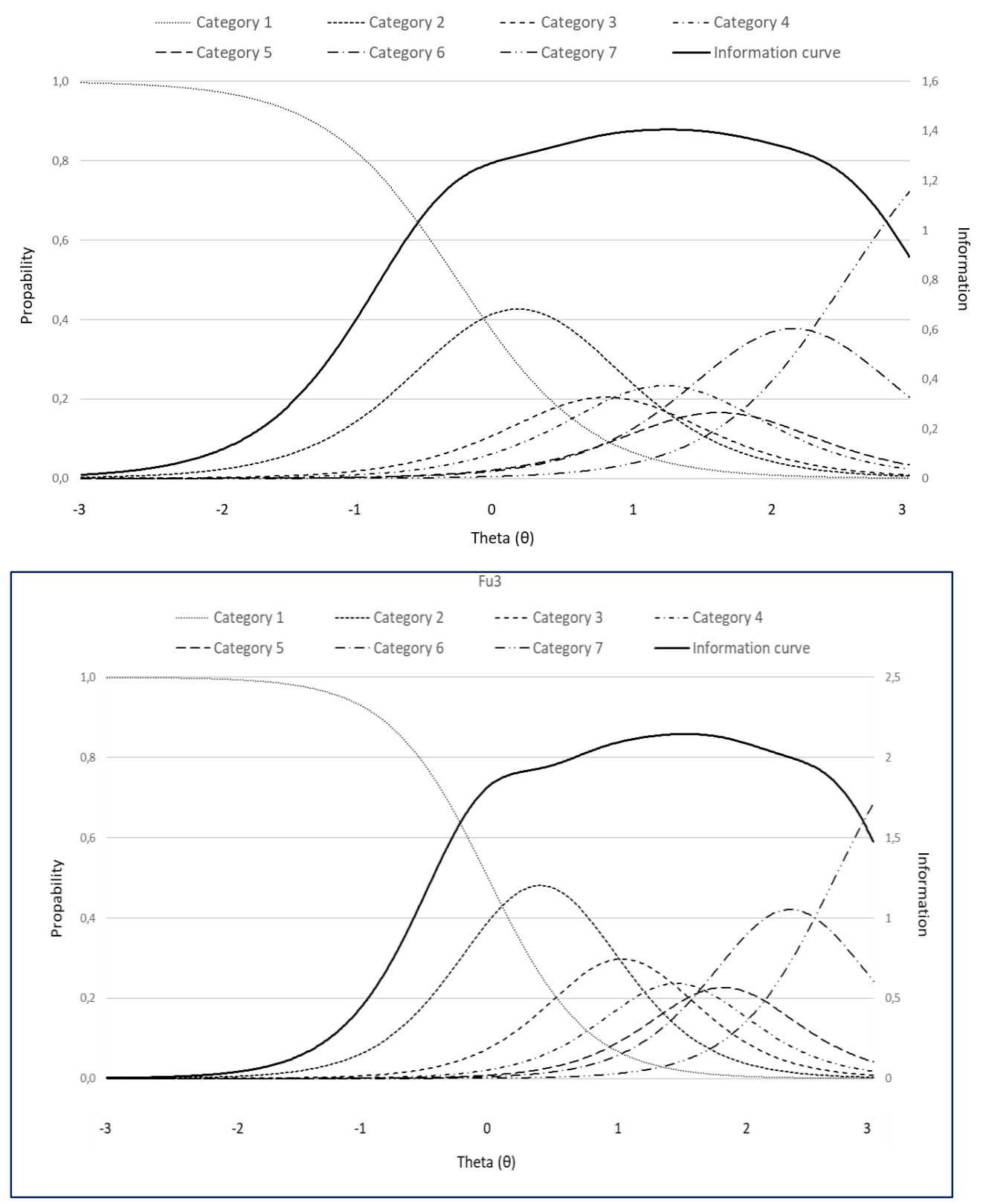

46
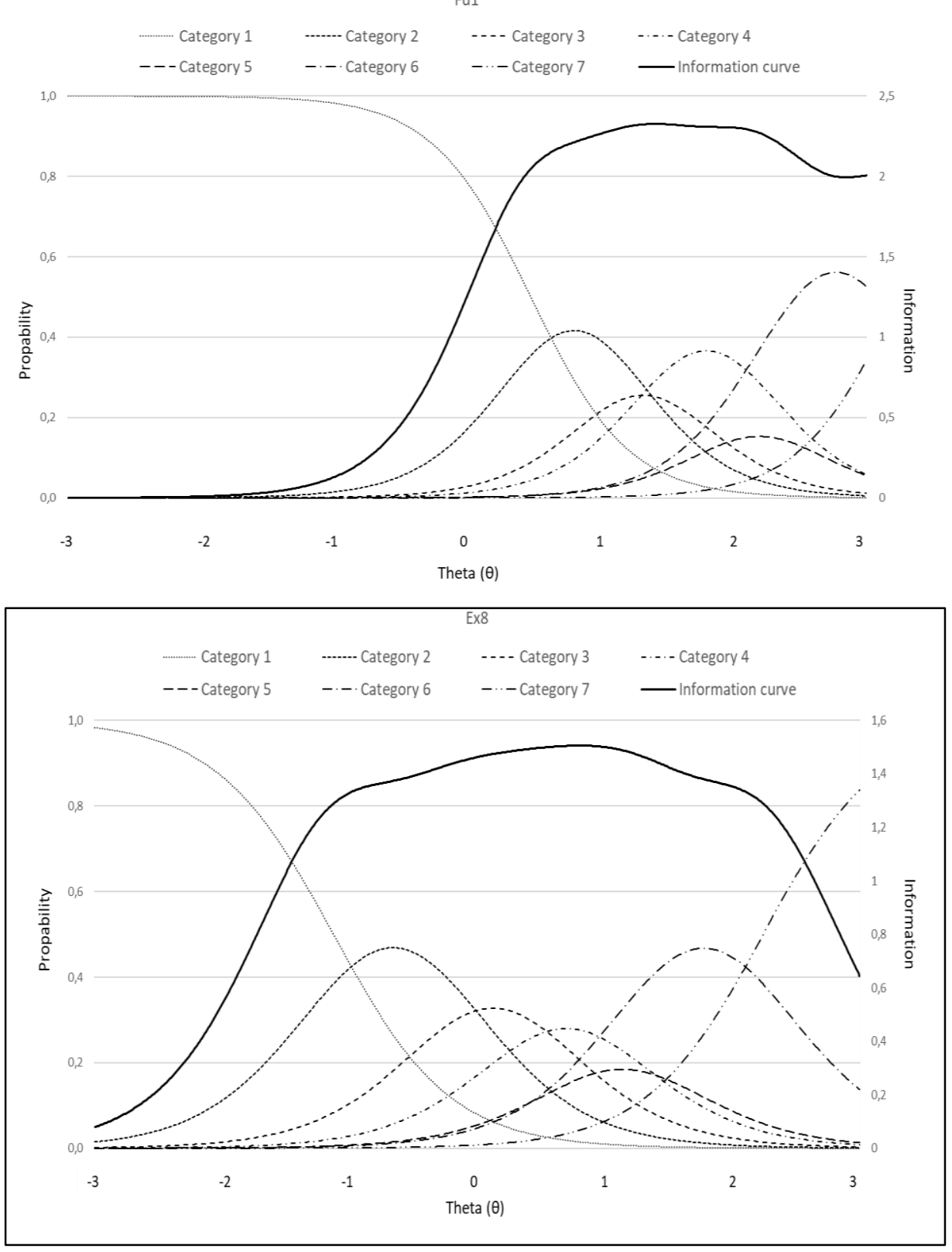
BRIEF PARENTAL BURNOUT SCALE
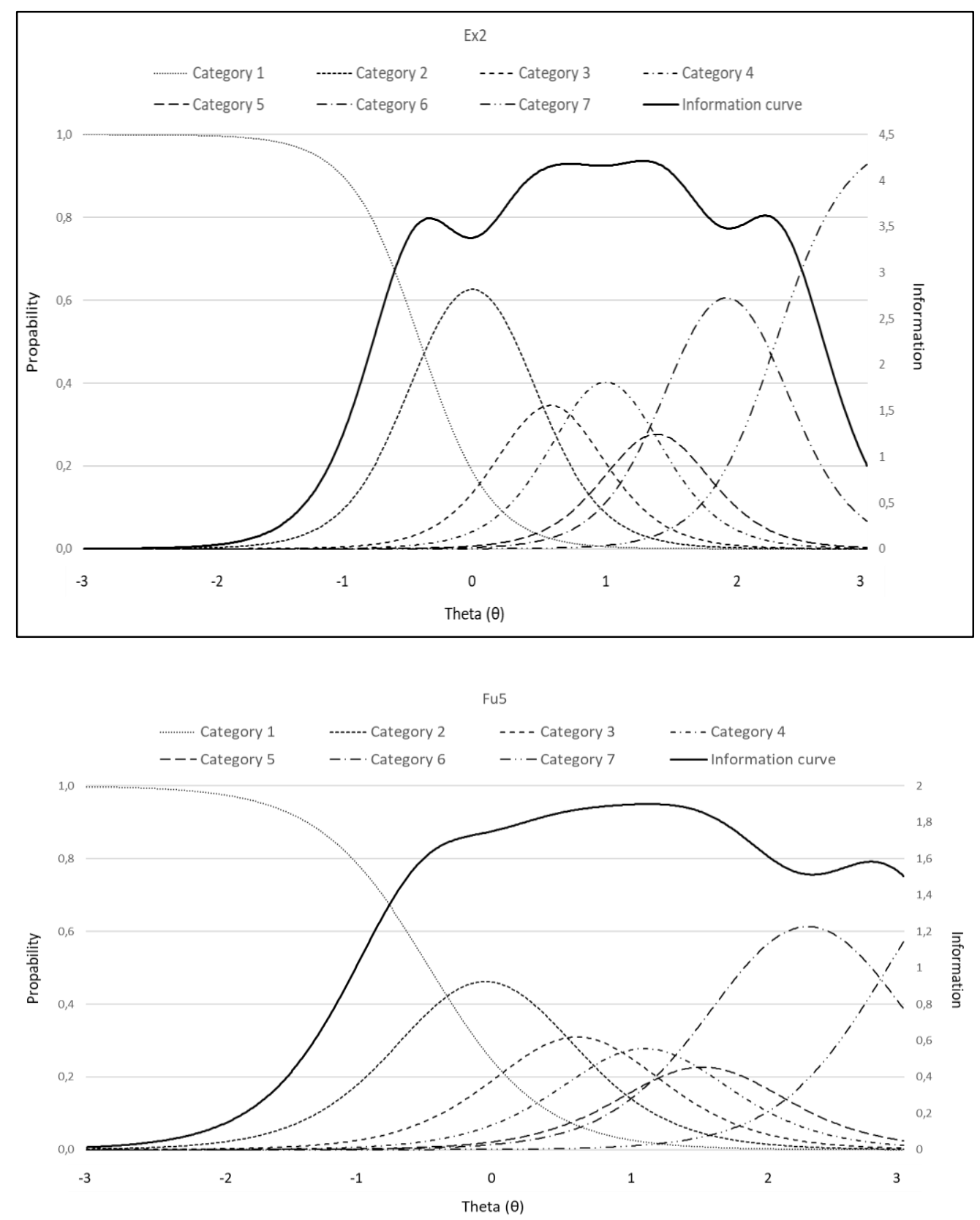

47
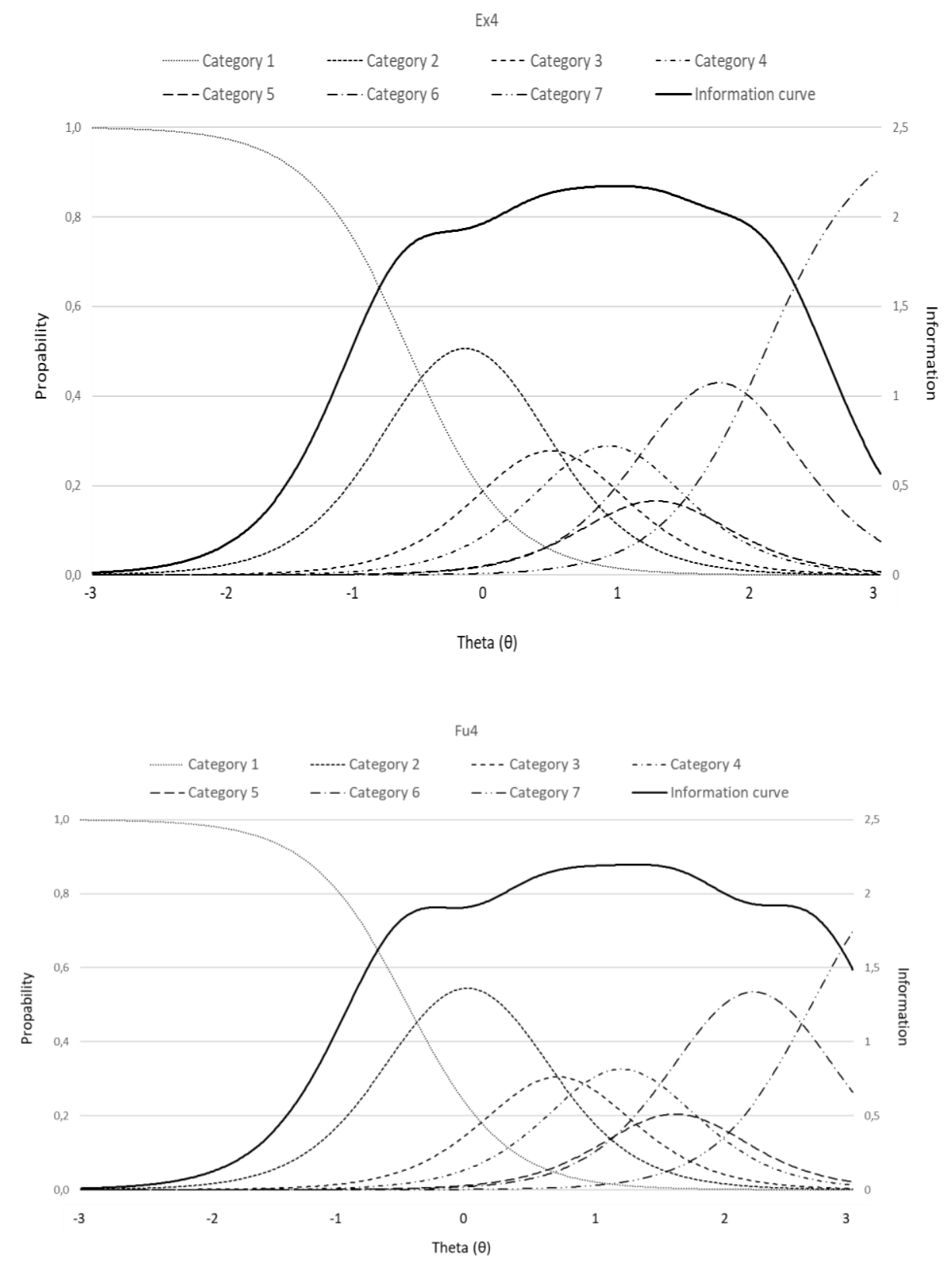
BRIEF PARENTAL BURNOUT SCALE
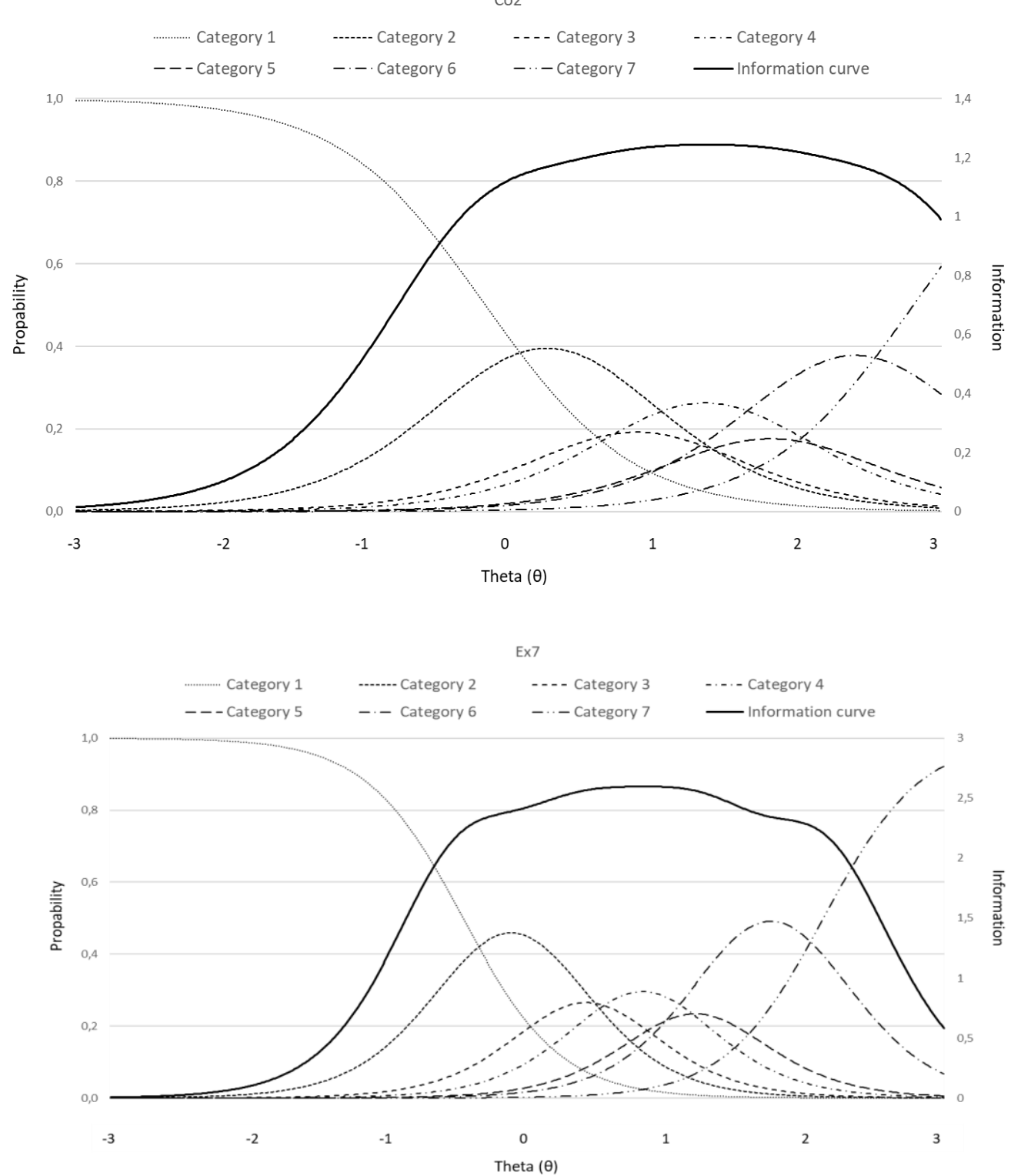

48

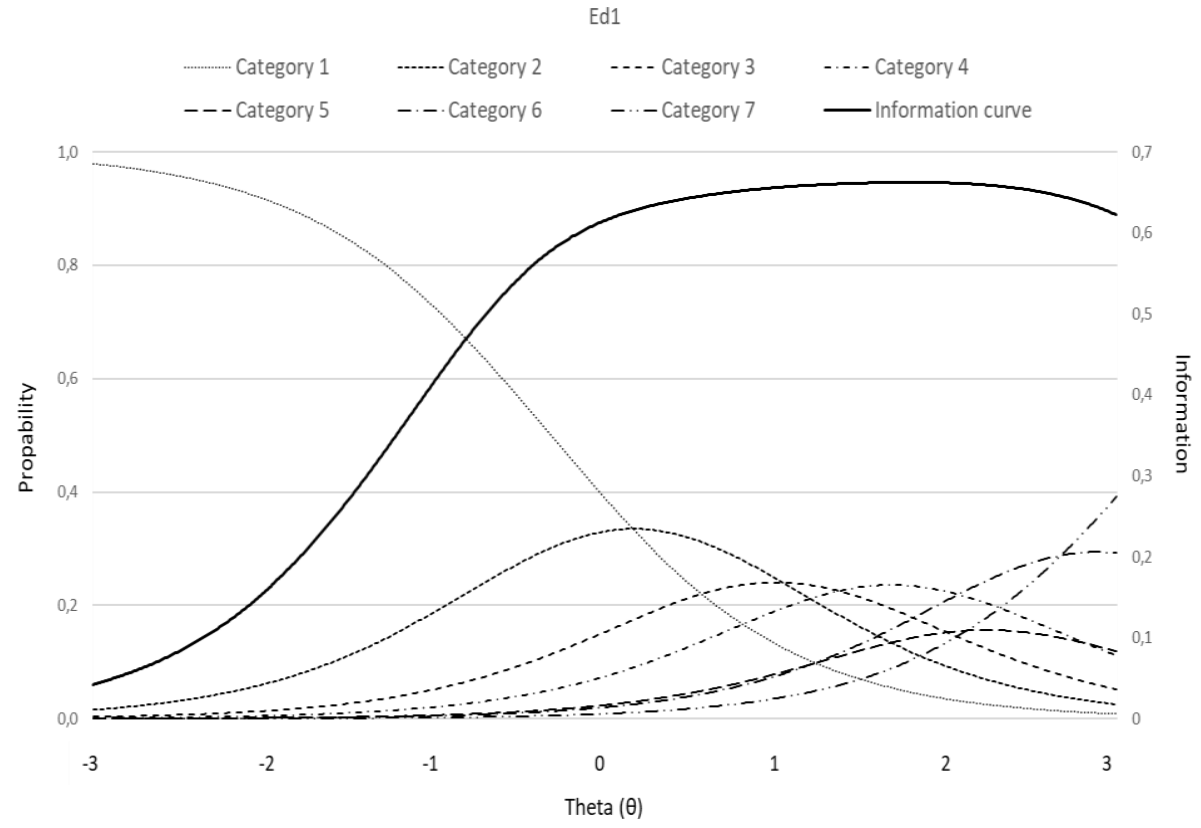

Fu2

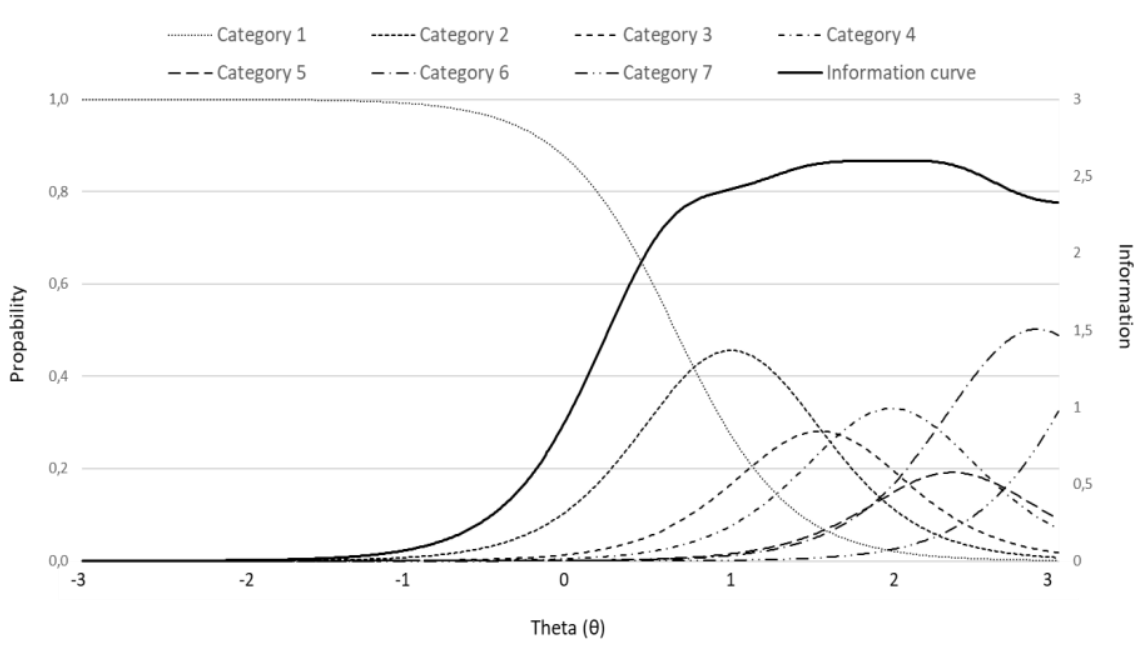


BRIEF PARENTAL BURNOUT SCALE
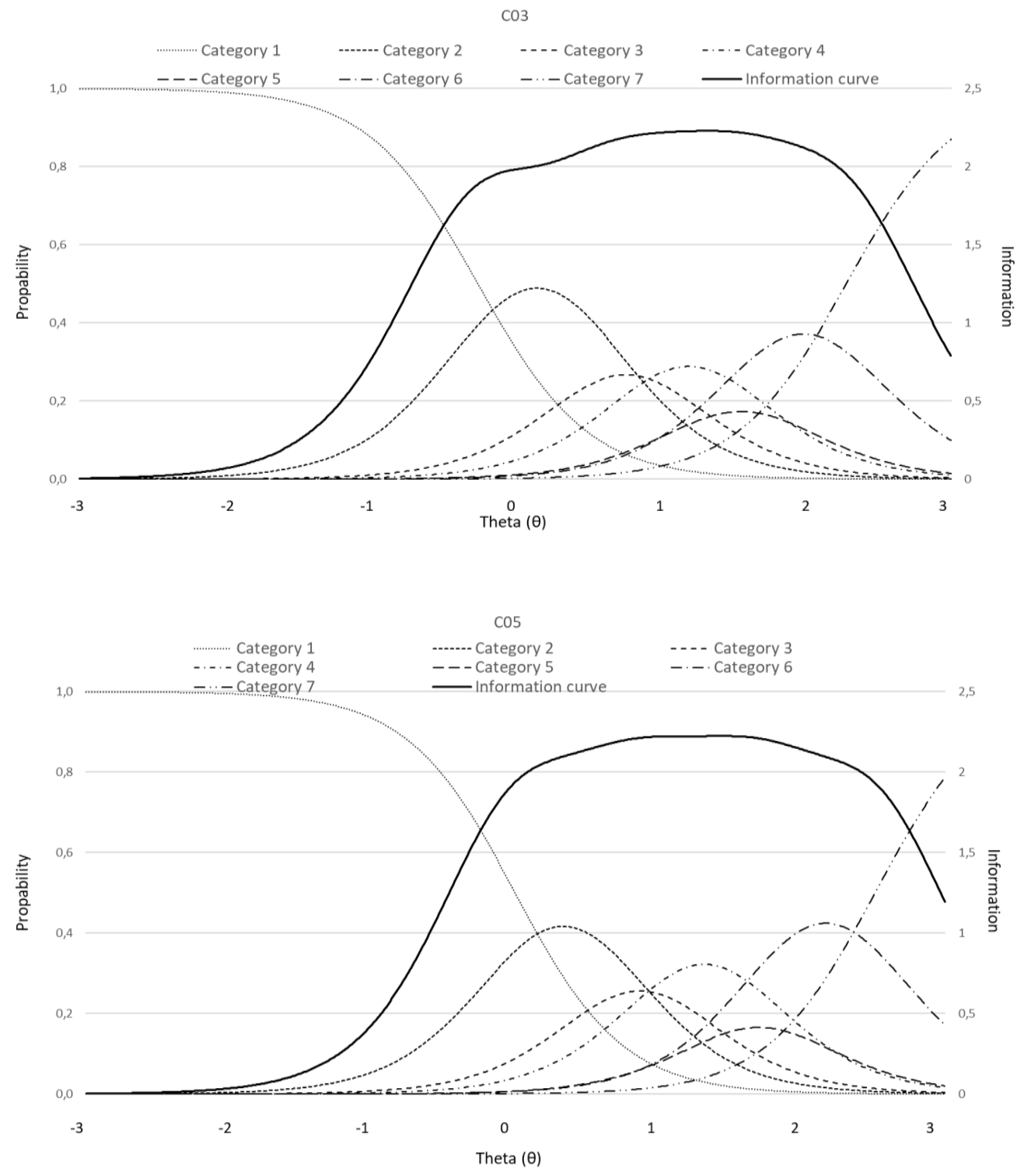

49
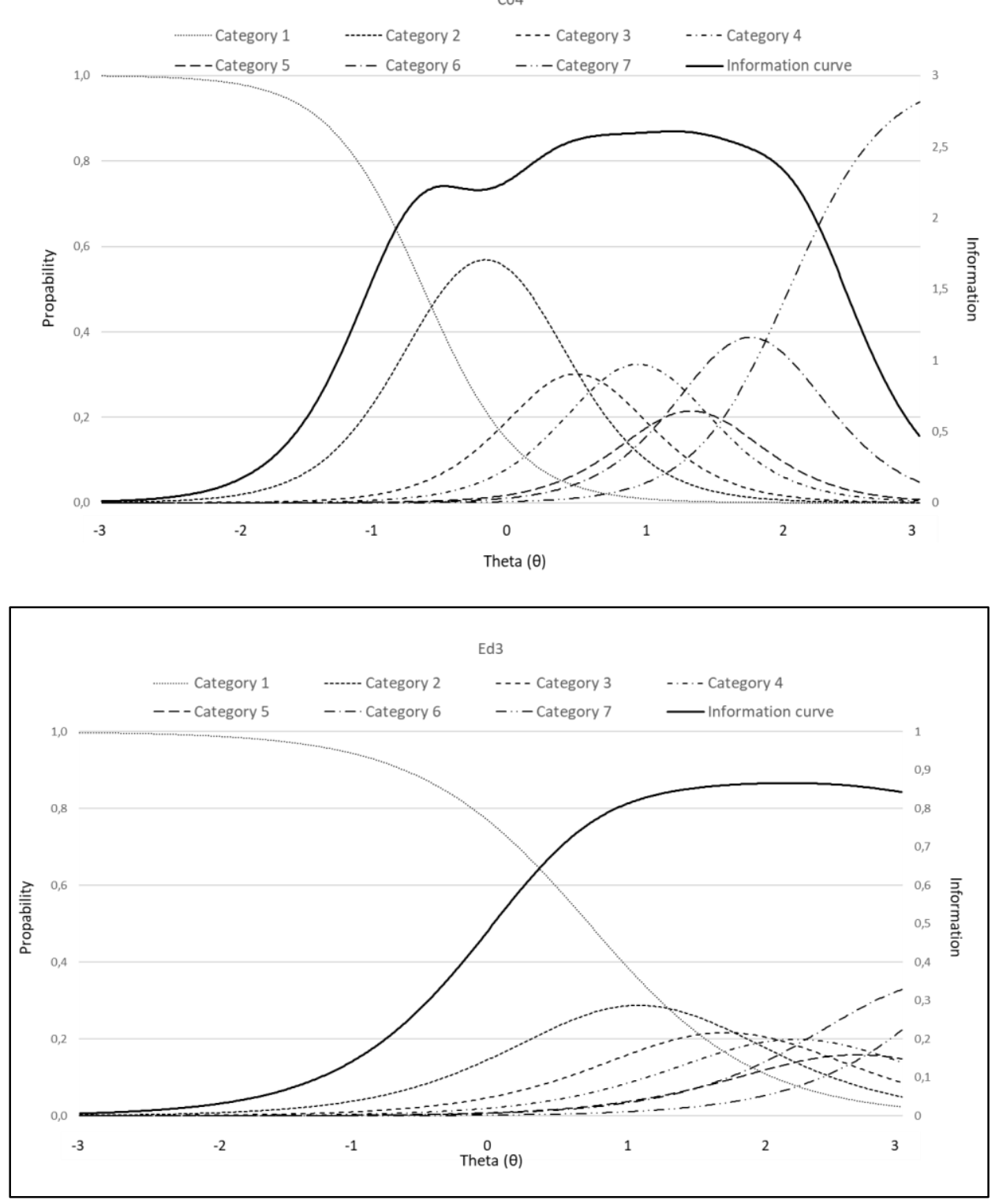

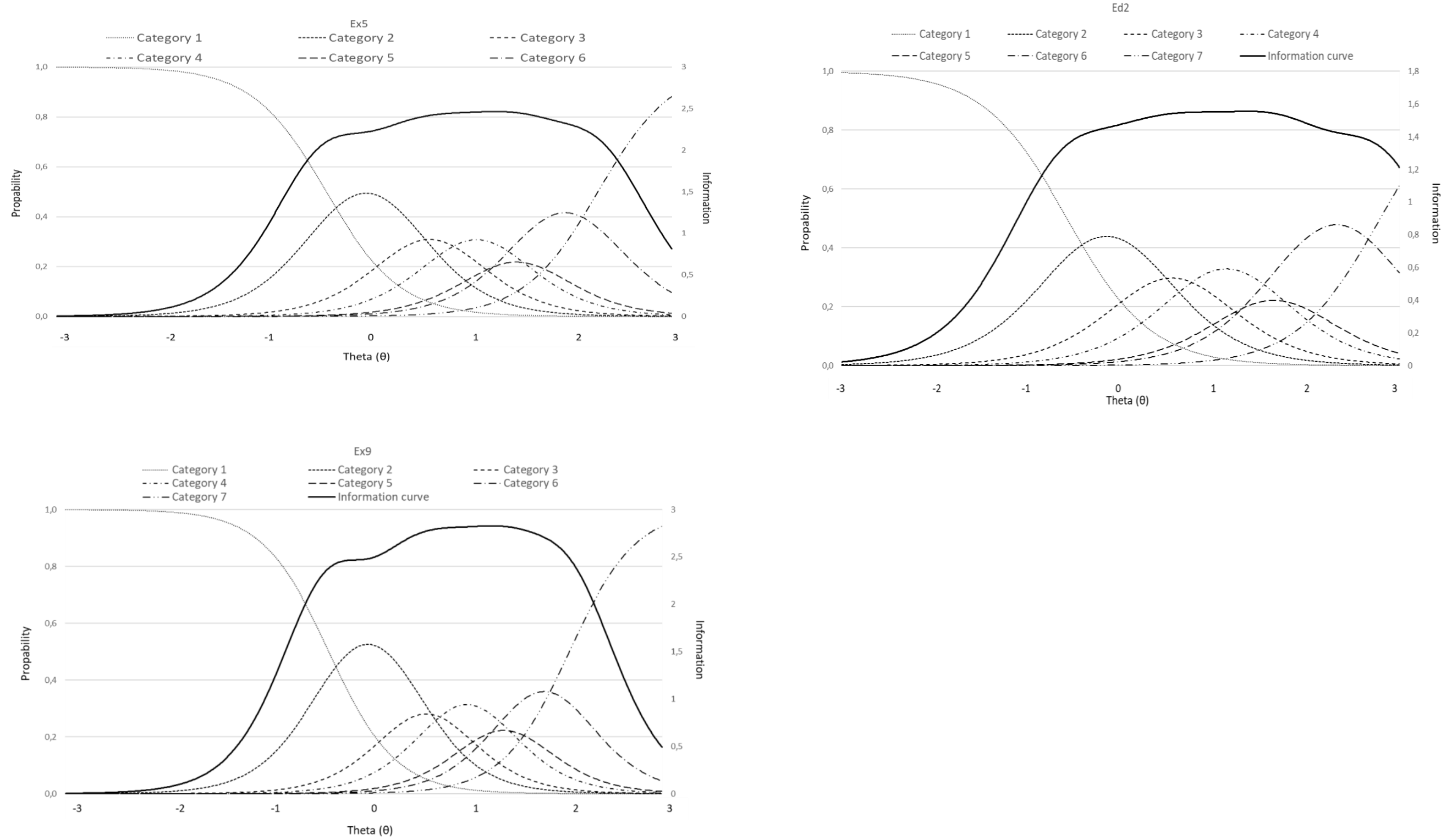